\title{
PREDICTION OF DISPLACEMENT AND STRESS FIELDS OF A NOTCHED PANEL WITH GEOMETRIC NONLINEARITY BY REDUCED ORDER MODELING
}

\author{
Ricardo Perez ${ }^{*}$ X. Q. Wang ${ }^{\dagger}$, and Marc P. Mignolet ${ }^{\ddagger}$ \\ Arizona State University, Tempe, AZ 85287-6106, USA \\ *Currently Postdoctoral Research Engineer, Universal Technology Corporation \\ ${ }^{\dagger}$ Associate Research Scientist, SEMTE, Faculties of Mechanical and Aerospace Engineering \\ ${ }^{\ddagger}$ Professor, SEMTE, Faculties of Mechanical and Aerospace Engineering
}

\begin{abstract}
The focus of this investigation is on a first assessment of the predictive capabilities of nonlinear geometric reduced order models for the prediction of the large displacement and stress fields of panels with localized geometric defects, the case of a notch serving to exemplify the analysis. It is first demonstrated that the reduced order model of the notched panel does indeed provide a close match of the displacement and stress fields obtained from full finite element analyses for moderately large static and dynamic responses (peak displacement of 2 and 4 thicknesses). As might be expected, the reduced order model of the virgin panel would also yield a close approximation of the displacement field but not of the stress one. These observations then lead to two "enrichment" techniques seeking to superpose the notch effects on the virgin panel stress field so that a reduced order model of the latter can be used. A very good prediction of the full finite element stresses, for both static and dynamic analyses, is achieved with both enrichments.
\end{abstract}




\section{INTRODUCTION}

Interest in the prediction of the dynamic response of thin panels undergoing "large" deformations (i.e., exhibiting geometric nonlinearity) has motivated the construction of reduced order models (ROMs) from finite element models generated using commercial codes (e.g. Nastran, Abaqus), see [1] for a recent review. This non-intrusive formulation allows for the relatively straightforward consideration of complex structural problems using tools that are routinely used in the industrial setting. The counterpart of these advantages is the unavailability of certain information and the uncertainty on the formulation implemented in the finite element modeling and response computation.

Notwithstanding the above difficulties, the ROM capabilities have progressed from applications to flat structures (see [2-9]), to moderately large motions of curved structures (see [10-14]). Further, the coupling of these nonlinear structural reduced order models with aerodynamics, either full or reduced order model has also been successfully demonstrated in [1517]. A similar coupling but of the structural dynamics and thermal aspects, the two in reduced order model format, has also been proposed and validated in [18-21]. In addition, validation studies with experiments have been carried out for different types of panels [3, 23-24]. The introduction of uncertainty in the reduced order model has finally been formulated and implemented [25,26].

The reduced order models developed in the above investigations are parametric, i.e. the form of the equations governing the generalized coordinates is fixed, linear in mass and damping operators with a stiffness operator exhibiting linear, quadratic, and cubic terms in all combinations of generalized coordinates as derived from finite deformation elasticity in the reference configuration (see review below). 
Panels represent a basic building block of wings and aircraft fuselage; however, smaller scales are also present. These scales may result from the structural design (e.g. fasteners) or may arise from damage (e.g. cracks, debonds) and are expected to have a localized effect in the stress field. In this light, the focus of the present investigation is on a first assessment of the predictive capabilities of reduced order models for panels that have a localized geometric defect, such as a notch which will be considered here. Two particular questions to be addressed here are:

(1) how well do reduced order models capture the stress distribution in the notch near-field, and

(2) how could the reduced order modeling process of the defect-free (or virgin) panel be employed?

For completeness, the derivation of the reduced order modeling strategy is first briefly reviewed.

\section{REDUCED ORDER MODELING}

\subsection{Reduced Order Model Form and Governing Equations}

The reduced order models considered here are based on a representation of the nonlinear geometric response in terms of a set of basis functions

$$
\mathbf{u}(t)=\sum_{n=1}^{M} q_{n}(t) \psi^{(n)}
$$

where $\mathbf{u}(t)$ represents the vector of displacements of the finite element degrees of freedom, $\boldsymbol{\psi}^{(n)}$ are specified, constant basis functions, and $q_{n}(t)$ are the time dependent generalized coordinates.

The reduced order modeling (ROM) procedure described here is achieved in the undeformed configuration $\Omega_{0}$ for which the field equations are (summation is implied over repeated indices)

$$
\frac{\partial}{\partial X_{k}}\left(F_{i j} S_{j k}\right)+\rho_{0} b_{i}^{0}=\rho_{0} \ddot{u}_{i}
$$


where $\mathbf{S}$ is the second Piola-Kirchhoff stress tensor, $\rho_{0}$ is the density with respect to the reference configuration, and $\mathbf{b}^{0}$ is the vector of body forces, all of which are assumed to depend on the position $\mathbf{X} \in \Omega_{0}$, [27,28]. Further, in Eq. (2), $\mathbf{F}$ denotes the deformation gradient tensor of components

$$
F_{i j}=\frac{\partial x_{i}}{\partial X_{j}}=\delta_{i j}+\frac{\partial u_{i}}{\partial X_{j}}
$$

where $\delta_{i j}$ is the Kronecker delta and $\mathbf{u}=\mathbf{x}-\mathbf{X}$ is the displacement vector, $\mathbf{x}$ being the position vector in the deformed configuration. An important aspect of the present formulation is that the material is assumed to be linear elastic in that $\mathbf{S}$ and $\mathbf{E}$ (the Green strain tensor) satisfy

$$
S_{i j}=C_{i j k l} E_{k l}
$$

where $\mathbf{C}$ is a fourth order elasticity tensor, function in general of the undeformed coordinates $\mathbf{X}$

To proceed, assume next the displacement field $u_{i}$ in the continuous structure in the form

$$
u_{i}(\mathbf{X}, t)=\sum_{n=1}^{M} q_{n}(t) U_{i}^{(n)}(\mathbf{X}) \quad i=1,2,3
$$

where $U_{i}^{(n)}(\mathbf{X})$ are specified, constant basis functions satisfying the boundary conditions also in the undeformed configuration. Equation (5) is the continuous space equivalent of the discrete, finite element model, representation of Eq. (1),

By introducing Eq. (5) in Eqs. (2)-(4) and imposing the condition that the error be orthogonal to the basis (Galerkin approach), a set of nonlinear ordinary differential equations for the generalized coordinates $q_{n}(t)$ can be obtained [8], they are the reduced order model equations

$$
M_{i j} \ddot{q}_{j}+D_{i j} \dot{q}_{j}+K_{i j}^{(1)} q_{j}+K_{i j l}^{(2)} q_{j} q_{l}+K_{i j l p}^{(3)} q_{j} q_{l} q_{p}=F_{i}
$$


where a linear damping term $D_{i j} \dot{q}_{j}$ has been added to collectively represent various dissipation mechanisms. Further, $M_{i j}$ denotes the elements of the mass matrix, $K_{i j}^{(1)}, K_{i j l}^{(2)}, K_{i j l p}^{(3)}$ are the linear, quadratic, and cubic stiffness coefficients and $F_{i}$ are the modal forces. Integral expressions for all coefficients of Eq. (6) are given in [8]. They can be used for their estimation (see [26]) but an indirect evaluation of many of these coefficients from the finite element model is also possible (see $[1,8,22,29])$ and is adopted here.

From the generalized coordinates, a complete solution of the problem and other quantities can then be evaluated. For example, any component of the second Piola-Kirchhoff stress tensor at any point can be expressed as

$$
S_{i j}=\bar{S}_{i j}+\hat{S}_{i j}^{(m)} q_{m}+\tilde{S}_{i j}^{(m, n)} q_{m} q_{n}
$$

where the coefficients $\bar{S}_{i j}, \hat{S}_{i j}^{(m)}$, and $\widetilde{S}_{i j}^{(m, n)}$ depend only on the point $\mathbf{X}$ considered and can be obtained non-intrusively from finite element computations [8].

\subsection{Basis Selection}

One of the key aspects of the reduced order modeling strategy is the selection of the basis functions $\psi^{(n)}$. A poor representation of the structural response within this basis will lead to a poor prediction of the response by the reduced order model. The basis is certainly expected to include the modes used for the corresponding linear problem (i.e. low frequency modes), but this is not sufficient in the nonlinear geometric regime. Indeed, the displacement fields of these linear modes for shell-like structures are primarily transverse/normal to the structure and thus do not capture accurately the in-plane/tangential displacements that take place when the deformations are no longer infinitesimal. For example, if the structure is completely clamped, transverse 
deformations imply a stretching (referred to as the membrane stretching, see [1,3,6] for discussion) of the structure. For cantilevered structures, this effect is reflected by a shortening of the domain occupied by the structure in the undeformed configuration. These in-plane/tangential displacements typically lead to a significant softening of the transverse motions through a nonlinear coupling effect and thus must be captured accurately to achieve a good prediction of the transverse displacements. This need will be demonstrated in the results section below.

A first approach to select a basis to model the in-plane/tangential displacements would be to use linear modes that are dominated by such motions but they are difficult to identify in complex structures. Alternatively, a dedicated basis can be developed [8] that rests on the following two observations:

(a) the in-plane/tangential motions to be captured exhibit high natural frequencies, much higher than the excitation bandwidth.

(b) the in-plane/tangential motions are primarily driven by the transverse motions through their nonlinear coupling as opposed to excited directly by the excitation.

The observation (a) implies that the in-plane/tangential motions take place quasi statically and thus the basis functions sought can be obtained from static analyses. From observation (b), it is further concluded that the loading in these analyses should excite primarily the modes from the linear basis which are transverse dominated. An intuitive approach (see [8] for further discussion) to achieve this condition is to rely on the linear situation. That is, the applied load vectors $\mathbf{F}_{F E}^{(m)}$ on the structural finite element model necessary to induce linear static responses of the form

$$
\mathbf{u}^{(m)}=\sum_{i} \alpha_{i}^{(m)} \psi^{(i)}
$$


are

$$
\mathbf{F}_{F E}^{(m)}=\sum_{i} \alpha_{i}^{(m)} \mathbf{K}_{F E}^{(1)} \boldsymbol{\psi}^{(i)}
$$

where $\alpha_{i}^{(m)}$ are coefficients to be chosen with $m$ denoting the load case number and $\mathbf{K}_{F E}^{(1)}$ is the linear finite element stiffness matrix of the structure.

The nonlinear geometric static response of the structure $\mathbf{u}_{N L}^{(m)}$ to the loading of Eq. (9) is not exactly in the form of Eq. (8), a discrepancy vector $\boldsymbol{\delta}^{(m)}$ is observed as

$$
\boldsymbol{\delta}^{(m)}=\mathbf{u}_{N L}^{(m)}-\sum_{i} \beta_{i}^{(m)} \psi^{(i)}
$$

where $\beta_{i}^{(m)}$ are the projections of $\mathbf{u}_{N L}^{(m)}$ on the modes $\boldsymbol{\psi}^{(i)}$. The discrepancies $\boldsymbol{\delta}^{(m)}$ represent the information on which the additional basis functions are to be constructed. This step is achieved through a proper orthogonal decomposition (POD) of an ensemble of discrepancy vectors obtained for various linear combinations of the modes $\psi^{(i)}$ in Eq. (8). In fact, a detailed discussion of the linear combinations to be used is presented in [8] but, in all validations carried out, it has been sufficient to consider the cases

$$
\mathbf{F}_{F E}^{(m)}=\alpha_{i}^{(m)} \mathbf{K}_{F E}^{(1)} \boldsymbol{\psi}^{(i)} \quad i=\text { dominant mode }
$$

and

$$
\mathbf{F}_{F E}^{(m)}=\frac{\alpha_{i}^{(m)}}{2} \mathbf{K}_{F E}^{(1)}\left[\psi^{(i)}+\psi^{(j)}\right] i=\text { dominant mode, } j \neq i
$$

where a "dominant” mode is loosely defined as one expected to provide a large component of the panel response to the physical loading. The ensemble of loading cases considered is formed by selecting several values of $\alpha_{i}^{(m)}$ for each dominant mode in Eq. (11) and also for each mode 
$j \neq i$ in Eq. (12). Further, note that both positive and negative values of $\alpha_{i}^{(m)}$ are suggested and that their magnitudes should be such that the corresponding displacement fields $\mathbf{u}_{N L}^{(m)}$ range from near linear cases to some exhibiting a strong nonlinearity.

The basis functions resulting from this process are the "dual modes" [8] to be appended to the linear modes to form the basis. The appropriateness of these modes can be assessed as in [29] by comparing the displacements induced by the loads of Eqs (11) and (12) and by physical (static or dynamic) loads in sections of the $N$ dimensional space, where $N$ denotes the number of degrees of freedom of the structure. This comparison will be successfully carried out in the reduced order model construction section.

\subsection{Overall Modeling Process}

The overall modeling process is summarized in the flow chart of Fig. 1. The finite element model is first used to produce the linear modes (step 1) through an eigensolution. Combinations of these modes (step 2) are constructed according to Eqs (11) and (12) to produce the load cases necessary to generate the dual modes. These load cases are applied (step 3) to the finite element model of which the static nonlinear deflections are determined (step 4). These deflections are projected on the linear modes and the corresponding discrepancies $\boldsymbol{\delta}^{(m)}$ generated (step 5), see Eq. (10), and processed in a proper orthogonal decomposition (step 6) to generate the dual modes. These modes are finally added to the linear modes (step 7) to form the final basis. The process is completed (step 8) by the evaluation of the stiffness coefficients according to one of the methodologies described in [1,8,22,29]. The imposed displacement method of [22] as modified in $[4,5,8]$ was selected here. 
In the present effort, the notation ROM $m \mathrm{Tn} n$ will be used to refer to a reduced order model constructed as in Fig. 1 using $m$ linear modes (mostly transverse, thus "T") and $n$ dual modes (“D”).

\section{MODELS FOR VALIDATION: NOTCHED AND VIRGIN PANEL MODELS}

A beam-like panel with the properties given in Table 1 was considered here with and without notch. The panel was assumed to have clamped-clamped boundary conditions and to be subjected to a uniform pressure of varying magnitude.

The notched beam exhibited a rounded notch of length equal to one-fourth of the beam thickness placed at 30\% of the length of the beam and along its entire width as shown in Figs. 2 and 3. Clearly, plane stress conditions cannot be assumed to exist on the $x$-z plane since it is not a thin membrane. On the other hand, plane strain conditions do not exist since this is not a long body problem. Therefore, the structure was discretized throughout its entire domain with 8-node brick elements (CHEXA in NX/Nastran), and 14 such elements were used along the width of the beam. Along the length, the notched beam was divided in different parts, as shown in Fig. 3, to capture the local effects of the notch without excessive meshing away from that zone. Away from the notch (parts (a) and (c) in Fig 2), 4 elements were used through the thickness of the beam and a uniform division along the length was performed with 20 elements for part (a) and 47 elements for part (c). The finer meshing around the notch (part (b) in Fig. 2) is shown in Fig. 3(a). Note further from this figure that the notch is rounded to avoid any plasticity in its vicinity, which is not considered in this first effort. The purpose of this work being on the validation of the reduced order modeling strategy in comparison with the corresponding finite element predictions, a very fine mesh was not necessary and thus was avoided to accelerate the 
computations.

A virgin beam model was also considered and, for ease of comparison, its meshing was selected to be identical to the one of the notched beam but with the notch filled with CHEXA elements, see Fig. 3(b), and with the same material properties, see Table 1. Reduced order models of the two beams were constructed as discussed in Section 2 and Fig. 1.

\section{REDUCED ORDER BASIS: NOTCHED AND VIRGIN PANELS}

It was first of interest to determine and compare the basis functions of the reduced order models, i.e., the transverse and duals modes, of the virgin and notched beams to assess the effects, local and/or global, of the notch.

The transverse linear modes were first investigated and were obtained, for the two beams, from a normal modes solution in NX/Nastran (SOL 103). The natural frequencies of the first 4 symmetric modes of the two beams are shown in Table 2. As expected, given the small size of the notch, its effect on the first few natural frequencies is very small.

The transverse and in-plane (along the length of the beam) components of the first mode shape along one of the top edges of the beams $(y=0, z=h)$ are shown in Figs. 4-5. The first observation to be drawn is that the notch does not affect noticeably the transverse displacements, but appears to induce a sharp peak in its in-plane counterpart. However, this peak is an artifact of the geometry, i.e., the notched beam data presented includes the displacement at the nodes along the flat edge of the beam but also those along the faces of the notch. Since these points are much closer to the neutral axis of the beam, their in-plane displacements are expected to be smaller as seen in Fig. 6. Plotting the same nodal displacements for the virgin beam, see Figs. 7-8, confirms this explanation of the peak. 
The linear modes of the structure only represent one part of the basis, modeling primarily the transverse displacements, while the dual modes (see Section 2.2 and $[8,9,14]$ ) capture the nonlinearly induced in-plane motions. In this light, it was also desired to assess the effects of the notch on these dual modes. The 4 dual modes corresponding to the 4 linear modes were created using Eqs (11)-(12) with mode 1 dominant, for both notched and virgin beams. The POD-based dual mode construction procedure highlighted above (see [8] for full details) was performed for the data obtained for mode 1 alone and each of the 3 combinations of mode 1 and another of the 3 largest responding modes. In each of these 4 situations, 10 different loading factors $\alpha_{i}^{(m)}$ were used, half positive and half negative, and leading to peak deflections ranging from 1 to approximately 4.4 skin panel thicknesses. The remainders of these 40 deflections, after projection on the 4 linear modes identified above, were analyzed by POD.

Shown in Figs. 9-10 are the transverse and in-plane components of the first (dominant) dual. Note that the notch is most present in the transverse component of the dual mode, see Fig. 9, which is quite different for the notched and virgin beams. In particular, note for the former the presence of a large, broad (as measured by the width of the notch) peak at the location of the notch. On the contrary, the in-plane components of this dual mode are almost unaffected by the notch, see Fig. 10. The most noticeable difference in the in-plane displacement is a jump occurring at the location of the notch, as seen in Fig. 11. Similar observations were drawn for the other 3 dual modes.

Before proceeding with detailed validations of the reduced order models, it is desirable to assess the adequacy of the dual modes to represent the in-plane displacements induced by the large transverse motions. To this end, it was proposed in [29] to compare the part of the threedimensional space occupied by three representative displacements obtained in various finite 
element computations to the similar space generated by the dual modes. In the present example, these three displacements were selected as the transverse displacement at the midpoint of the beam and the transverse and in-plane displacements at the notch tip. The data from the full finite element static analyses under uniform loads (see section 5 below) and snapshots of the corresponding dynamic response (see section 6) were used to generate these three-dimensional plots for both virgin and notched beam, see Fig. 12.

Also shown on these figures are the three displacement values induced by the loadings of Eq. (11) for the 1-1 dual and Eq. (12) for the combination of modes 1 and 2 (i.e. the 1-2 dual) for

various values of the coefficients $\alpha_{i}^{(m)}$. These cases closely occupy the same space as both static and dynamic physical loads and thus they can efficiently be used in the construction of the basis, dual modes specifically, for the representation of the full nonlinear response. Note as well that the displacements induced by the loading of Eq. (11) for the 2-2 dual do not occupy the same space as the other ones and thus is not a good candidate for the basis, i.e. its inclusion in the basis would not be detrimental but would not be very beneficial either. This observation is indeed expected as mode 2 is not a dominant mode, i.e. it does not or rarely does (in the dynamic case) represent the largest component of the response. This finding confirms the selection strategy of the modes, dominant or not, in Eq. (11) and (12) and the construction of the duals as in section 2.2.

\section{STATIC VALIDATION: DISPLACEMENT AND STRESS FIELDS}

Using the 4 linear and 4 dual modes analyzed above, reduced order models were built for both notched and virgin beams. To assess the adequacy of these reduced order models and assess the effects of the notch, the beams were loaded with uniform pressure acting on the bottom 
surface. Two different pressures were chosen, $2.6 \mathrm{kPa}$ and $17 \mathrm{kPa}$, which led to peak transverse displacements of approximately 2 and 4 beam thicknesses, well within the nonlinear range. The static responses were computed with the reduced order model as well as by a nonlinear NX/Nastran analysis (SOL 106). Shown in Figs. 13-16 is a comparison of the predicted transverse and in-plane displacements at the beam's upper and lower edges. Clearly, the matching is excellent for both transverse and in-plane displacements, even in the direct vicinity of the notch (see Fig. 17). Also shown in Figs 13 and 14 are the predictions obtained from the reduced order including only the 4 linear modes (i.e. with 0 duals and curves "ROM 4T0D"). This model clearly gives a poor prediction of the response demonstrating the need to account for the in-plane displacement fields, e.g. through the dual modes discussed above.

In Figs. 17-18, the response of the virgin beam was plotted at the same node locations as the response of the notched beam. Interestingly, the reduced order model of the virgin beam does an excellent job in capturing the in-plane displacement field in the notch region.

The static response of the beam to pressures equal to $-2.6 \mathrm{kPa}$ and $-17 \mathrm{kPa}$, applied on the bottom surface of the beam, and leading to peak transverse displacements of -2 and -4 beam thicknesses was also investigated [30] but is not shown here for brevity. The matching of the reduced order model and Nastran predictions was found excellent for both cases, similar to the one shown on Figs 13-18.

Shown in Table 3 is a summary of the prediction errors for the three displacement components. The prediction errors were computed as the norm of the difference between the NX/Nastran and ROM predictions divided by the norm of the NX/Nastran results and are shown for the ROMs of the notched and virgin beams. Clearly, the linear modes chosen to represent the transverse displacements, along with the modeling of the in-plane displacements by the duals, 
result in a very good matching of the transverse and dominant in-plane (T1) components with respect to NX/Nastran.

The previous results have demonstrated that the reduced order model of the notched beam is able to capture accurately the displacement field of this beam, thereby extending published validation cases, see [1] for review. Furthermore, it has been observed, not too unexpectedly, that the displacement fields of the notched and virgin beams are indeed very close to each other, suggesting that the latter could be used for the prediction of the response of the former.

Before any such connection can be established, however, it is necessary to assess the capability of the notched beam reduced order model to capture the stress distribution of this beam. To this end, shown in Figs 19-20 are the dominant stresses $S_{x x}$ along the top edge of the beam $(y=0, z=h)$, as computed by the reduced order model of the notched beam, see Eq. (7), and by NX/Nastran nonlinear (curves "NX/Nastran (Notched)") for all loading cases analyzed above. Clearly, the agreement is very good to excellent, even in the notch near field (and for negative loads, see [30]), as seen from the results in Tables 3-4. It is thus concluded from these validation

cases that the nonlinear geometric reduced order modeling technique developed in prior investigations is also applicable to notched panels for the prediction of both their displacement and stress fields.

\section{DYNAMIC DISPLACEMENT AND STRESS FIELDS}

Lastly, a dynamic transverse loading was added and the response computed in NX/Nastran SOL 601. The beam was subjected to a uniform pressure on its bottom surface varying randomly in time as a white noise band-limited process in the frequency range $[0,1042 \mathrm{~Hz}]$ to simulate an acoustic loading of overall sound pressure level $(O A S P L)$ of $147 \mathrm{~dB}$. Furthermore, to permit a 
close comparison between the NX/Nastran and ROM results, a simple Rayleigh damping model was adopted, i.e. for which the damping matrix is $D=\alpha M+\beta K$ with $\alpha=12.838 / \mathrm{s}$ and $\beta=2.061 \mathrm{E}$ 6s. This selection led to damping ratios between $0.5 \%$ and $1.3 \%$ for all four transverse modes in the excitation band. The time histories computed from the reduced order model were obtained with a Newmark- $\beta$ solver with the resulting nonlinear algebraic equations solved using a fixed-point algorithm. A time step of $4 \mathrm{E}-5 \mathrm{~s}$ was used for these computations. The peak transverse displacement observed at the center of the beam was of 3.06 beam thicknesses while its standard deviation was 1.23 beam thickness indicating that the response of the beam is well within the nonlinear geometric regime.

The power spectra of the transverse displacement at the middle of the beam and of the inplane displacement at the beam quarter point, both at the upper edge (i.e., $y=0, z=h$ ) are shown in Figs. 21-23. Clearly, based on the matching of the power spectral density of the NX/Nastran results, the ROMs of the notched beam and of the virgin beam match very well the dynamics of the beam. Interestingly, the matching of the power spectrum of the in-plane displacement along the T1 direction at the notch tip is very good as well, even for the ROM of the virgin beam.

The power spectral density of the dominant $S_{x x}$ element stresses, at different locations along the beam are shown in Figs. 24-26. Away from the notch, the power spectrum of both ROMs match NX/Nastran. Figure 26 shows clearly the amplification of the stress field at the notch. The power spectrum corresponding to the ROM of the notched beam matches its NX/Nastran counterpart very well.

\section{STRESS FIELD LOCAL ENRICHMENT}

\subsection{Motivation}

The findings from the previous section provide a framework to carry out dynamic simulations of the notched beam at a much reduced computational cost than a full finite element 
analysis. Yet, the reduced order model, in both its basis and its coefficients, depends on the notch geometry. This property is unfortunate in certain applications in which this geometry may be variable, e.g. when considering the notch as an uncertain defect or when envisioning the use of the reduced order model for crack propagation. For such analyses, it would be highly desirable to rely on a reference geometry, most simply the virgin beam, and enrich the solution by an extra component accounting for the existence and geometry of the crack as opposed to building a new reduced order model for every new notch geometry.

The loading considered in the present validation cases, and representative of the applied loads on panels, leads primarily to bending and stretching (from the nonlinear effects) and thus a mode I deformation is dominant. Accordingly, it is proposed here to estimate the stress in the neighborhood of the notch by adding to the virgin beam stress distribution induced by the external loading a term that accounts for the presence of the notch, i.e.

$$
S_{x x}^{(e n r)}(x, y, z, t)=S_{x x}^{(V R O M)}(x, y, z, t)+\Delta S_{x x}(x, y, z, t) \text { for }(x, y, z) \in[\hat{x}-2 h, \hat{x}+2 h] \times[0, w] \times[0, h]
$$

where $\hat{x}$ is the axial location of the notch and $S_{x x}^{(e n r)}(x, y, z, t)$ is the resulting (enriched) stress prediction for the notched beam at location $(x, y, z)$ and time $t$. Further, $S_{x x}^{(V R O M)}(x, y, z, t)$ is its counterpart obtained from the ROM of the virgin beam and $\Delta S_{x x}(x, y, z, t)$ is one of the stress enrichments described below.

Following a stress intensity factor perspective, it is suggested that this term be computed as the increment of stress induced by the notch for a loading corresponding to the in-plane stress distribution of the virgin beam in the vicinity of the notch (taken here at a distance of 2 thicknesses). Further, this enrichment term will be computed in a linear static analysis. 
Effectively, this approach replaces the stress distribution of the virgin beam in the vicinity of the notch by a stress distribution of the notched beam that smoothly connects to the virgin far-field behavior.

Two separate versions of this strategy were considered and assessed on the notched beam from previous sections. The first one focuses solely on the interaction of the beam stretching and the notch as if the stress distribution through the thickness of the virgin beam was constant, approximated by its value at 2 thicknesses away from the notch on the top surface of the beam, i.e. $S_{X X}^{(V R O M)}(\hat{x}+2 h, w / 2, h, t)$. That is, $\Delta S_{x x}(x, y, z, t)$ will be estimated as

$$
\Delta S_{x x}(x, y, z, t)=S_{x x}^{(V R O M)}(\hat{x}+2 h, w / 2, h, t)\left(\frac{\tilde{S}_{x x}^{(N L i n)}(x, y, z)}{\tilde{S}_{x x}^{(V L i n)}(x, y, z)}-1\right)
$$

where $\tilde{S}_{X X}^{(N \operatorname{Lin})}(x, y, z)$ is the stress at location $(x, y, z)$ induced linearly by a unit uniform axial pressure applied at axial location $\hat{x}+2 h$ to the notched beam and $\tilde{S}_{x x}^{(V \operatorname{Lin})}(x, y, z)$ is its counterpart for the virgin beam both computed from their respective linear finite element models. The term in parentheses in Eq. (14) is effectively the stress intensity factor due to the notch.

The enrichment formula of Eq. (14) is very simple but it neglects the effects due to bending, i.e. a mostly linear variation of the stress through thickness, and possibly through width variations. A second stress enrichment scheme was considered which addresses those factors. Specifically, the notched and virgin beams were subjected at location $\hat{x}+2 h$ to an axial pressure matching exactly the stress $S_{x x}^{(V R O M)}(\hat{x}+2 h, y, z, t)$ predicted by the virgin beam ROM. Linear quasi-static computations with this loading led to the stresses $\hat{S}_{x x}^{(N \operatorname{Lin})}(x, y, z, t)$ and $\hat{S}_{x x}^{(V \operatorname{Lin})}(x, y, z, t)$ for the notched and virgin beams respectively in the neighborhood of the notch. 
The second proposed enrichment $\Delta S_{x x}(x, y, z, t)$ is then the difference between these two stress fields, i.e.

$$
\Delta S_{X X}(x, y, z, t)=\hat{S}_{X X}^{(N \operatorname{Lin})}(x, y, z, t)-\hat{S}_{X X}^{(V \operatorname{Lin})}(x, y, z, t) .
$$

\subsection{Validation of Stress Enrichment: Static Loading}

Shown in Table 4 are the peak stresses at the notch, as computed by the reduced order model of the virgin beam with the two enrichments that were previously described. Clearly, the agreement is very good for the cases shown. Less accurate predictions were obtained for negative pressures that promote the closing of the notch and thus have smaller stress intensity factors, see [30] for details. The stress distributions along the beam corresponding to the first enrichment strategy (the second one gave similar results) and cases 1 and 2 are shown in Figs. (19) and (20) (curves "ROM 4T4D (Virgin/Virgin) + Stress Enrichment"). The matching with the NX/Nastran predictions from the notched beam are clearly very good. Note that the stresses of the NX/Nastran analysis for the virgin beam were enriched as well to assess the accuracy of the enrichment procedure independently of the reduced order model. The enriched NX/Nastran stress results agree very well with the corresponding predictions on the notched beam as can be seen from Figs (19) and (20) (curves "NX/Nastran (Virgin) + Stress Enrichment") for the first enrichment strategy. The second one gave similar results.

\subsection{Validation of Stress Enrichment: Dynamic Loading}

For completeness it was desired to assess the effect of the enrichments in a dynamic analysis. The dynamic loading described in section 6 was used. The power spectral density of the $S_{x x}$ element stresses, at different locations along the beam are shown in Figs. 27-30. Interestingly, 
both enrichments lead to almost identical stress results. As seen in Fig. 27, the addition of the two enrichment schemes to the ROM of the virgin beam, resulted in a good matching of NX/Nastran near the notch, even though the virgin predictions were significantly too low, see Fig. 26. Note that the spectra of Figs 27-30 include both phases of notch opening and notch closing displacements. Thus, the less accurate predictions obtained in the static case with a negative pressure (see Section 7.2 and [30]) do not affect significantly the overall prediction in the dynamic case.

\section{CONCLUSIONS}

The focus of this investigation was on a first assessment of the predictive capabilities of nonlinear reduced order models for panels with a localized defect, i.e. a stress "hot spot". An aluminum clamped-clamped beam with a notch placed at $30 \%$ of its length and of depth equal to a quarter of the thickness was considered as an example. As expected, the notch was found to have a negligible effect on the first few natural frequencies of the beam, but also on the corresponding mode shapes. In addition, only small notch-related effects could be detected on the in-plane component of the dual modes, which are basis functions constructed to capture the nonlinear transverse in-plane coupling occurring in large deformations. However, a large, rather broad peak was observed in the smaller transverse component of the dual modes of the notched beam which is absent on the corresponding plot for the virgin beam.

The displacement field induced by a uniform pressure on the beam large enough to induce nonlinearity, i.e. peak transverse displacements of the order of 2 and 4 thicknesses, was also found to be very weakly dependent on the notch. Further, this displacement field was shown to be well predicted by the reduced order models of both notched and virgin beams. 
In regards to the prediction of the stress field, it was found that the notch beam reduced order model was indeed able to capture accurately the stress distribution induced by the pressure loading. Excellent prediction of the displacements and stresses was also achieved under dynamic loading conditions.

Nevertheless, it was questioned whether a prediction based on the virgin beam reduced order model could also be used if appropriately "enriched" with the notched beam stress field in a superposition-like manner. Two enrichment options were assessed that rely on this stress field as obtained, in a linear finite element static analysis, from a notched beam subjected to the stress state induced on the virgin beam near the notch location. This methodology led to good to excellent predictions of the stress field near the notch for both static and dynamic excitations.

\section{ACKNOWLEDGEMENTS}

The financial support of this work by the grant FA9550-10-1-0080 from the Air Force Office of Scientific Research with Dr D. Stargel as grant monitor is gratefully acknowledged.

\section{REFERENCES}

[1] Mignolet, M.P., Przekop, A., Rizzi, S.A., and Spottswood, S.M., “A Review of Indirect/NonIntrusive Reduced Order Modeling of Nonlinear Geometric Structures,” Journal of Sound and Vibration, Vol. 332, No. 10, pp. 2437-2460, 2013.

[2] McEwan, M.I., Wright, J.R., Cooper, J.E., and Leung, A.Y.T., “A combined Modal/Finite Element Analysis Technique for the Dynamic Response of a Nonlinear Beam to Harmonic Excitation,” Journal of Sound and Vibration, Vol. 243, pp. 601-624, 2001. 
[3] Hollkamp, J.J., Gordon, R.W., and Spottswood, S.M., "Nonlinear Modal Models for Sonic Fatigue Response Prediction: A Comparison of Methods,” Journal of Sound and Vibration, Vol. 284, pp. 1145-1163, 2005.

[4] Mignolet, M.P., Radu, A.G., and Gao, X., "Validation of Reduced Order Modeling for the Prediction of the Response and Fatigue Life of Panels Subjected to Thermo-Acoustic Effects,” Proceedings of the 8th International Conference on Recent Advances in Structural Dynamics, Southampton, United Kingdom, Jul. 14-16, 2003.

[5] Radu, A., Yang, B., Kim, K., and Mignolet, M.P., "Prediction of the Dynamic Response and Fatigue Life of Panels Subjected to Thermo-Acoustic Loading,” Proceedings of the 45th Structures, Structural Dynamics, and Materials Conference, Palm Springs, California, Apr. 1922, 2004. Paper AIAA-2004-1557.

[6] Hollkamp, J.J., and Gordon, R.W., 2008, "Reduced-Order Models for Nonlinear Response Prediction: Implicit Condensation and Expansion,” Journal of Sound and Vibration, Vol. 318, pp. 1139-1153.

[7] Przekop, A., and Rizzi, S.A., “A Reduced Order Method for Predicting High Cycle Fatigue of Nonlinear Structures,” Computers and Structures, Vol. 84, No. 24-25, pp. 1606-1618, 2006.

[8] Kim, K., Radu, A.G., Wang, X.Q., and Mignolet, M.P., “Nonlinear Reduced Order Modeling of Isotropic and Functionally Graded Plates,” International Journal of Nonlinear Mechanics, Vol. 49, pp. 100-110, 2013.

[9] Kim, K., Khanna, V., Wang, X.Q., and Mignolet, M.P., “Nonlinear Reduced Order Modeling of Flat Cantilevered Structures,” Proceedings of the 50th Structures, Structural Dynamics, and Materials Conference, Palm Springs, California, May 4-7, 2009. AIAA Paper AIAA-2009-2492. 
[10] Przekop A., and Rizzi S.A., "Nonlinear Reduced Order Random Response Analysis of Structures with Shallow Curvature,” AIAA Journal Vol. 44 (8), pp. 1767-1778, 2006.

[11] Gordon R.W., and Hollkamp, J.J., "Reduced-Order Modeling of the Random Response of Curved Beams using Implicit Condensation,” AIAA-2006-1926, 2006.

[12] Spottswood, S.M., Hollkamp, J.J., and Eason, T.G., "On the Use of Reduced-Order Models for a Shallow Curved Beam Under Combined Loading," Proceedings of the 49th Structures, Structural Dynamics, and Materials Conference, Schaumburg, Illinois, Apr. 7-10, 2008. AIAA Paper AIAA-2008-1873.

[13] Przekop, A., and Rizzi, S.A., "Dynamic Snap-Through of Thin-Walled Structures by a Reduced-Order Method,” AIAA Journal, Vol. 45, No. 10, pp. 2510-2519, 2007.

[14] Spottswood, S.M., Eason, T.G., Wang, X.Q., and Mignolet, M.P., “Nonlinear Reduced Order Modeling of Curved Beams: A Comparison of Methods," Proceedings of the 50th Structures, Structural Dynamics, and Materials Conference, Palm Springs, California, May 4-7, 2009. AIAA Paper AIAA-2009-2433.

[15] Kim, K., Kim, Y.C., Mignolet, M.P., Liu, D.D., Chen, P.C., Lee, D.H., "Random Aeroelastic Response Due to Strong Hypersonic Unsteady-Wave/Shock Interaction with Acoustic Loads," Proceedings of the 48th Structures, Structural Dynamics, and Materials Conference, Honolulu, Hawaii, Apr. 23-26, 2007. AIAA Paper AIAA-2007-2014.

[16] Liu, D.D., Chen, P.C., Zhang, Z., Wang, Z., Yang, S., Lee, D.H., Mignolet, M.P., Kim, K., Liu, F., Lindsley, N., and Beran, P., "Continuous Dynamic Simulation of Nonlinear Aerodynamic/Nonlinear Structure Interaction (NANSI) for Morphing Wing Aeroelasticity,” Proceedings of the 50th Structures, Structural Dynamics, and Materials Conference, Palm Springs, California, May 4-7, 2009. AIAA Paper AIAA-2009-2572. 
[17] Liu, D.D., Wang, Z., Yang, S., Cai, C., Wang, X.Q., and Mignolet, M.P., “Nonlinear Aeroelastic Methodology for A Membrane-on-Ballute Model with Hypersonic Bow Shock,” Proceedings of the 50th Structures, Structural Dynamics, and Materials Conference, Palm Springs, California, May 4-7, 2009. AIAA Paper AIAA-2009-2363.

[18] Perez, R., Wang, X.Q., and Mignolet, M.P., “Nonlinear Reduced Order Models for Thermoelastodynamic Response of Isotropic and FGM Panels,” AIAA Journal, Vol. 49, pp. 630641, 2011.

[19] Perez, R., Wang, X.Q., and Mignolet, M.P., “Steady and Unsteady Nonlinear Thermoelastodynamic Response of Panels by Reduced Order Models,” Proceedings of the 51th Structures, Structural Dynamics, and Materials Conference, Orlando, Florida, April 12-15, 2010. AIAA Paper AIAA-2010-2724.

[20] Matney, Perez, R., and Mignolet, M.P., "Nonlinear Unsteady Thermoelastodynamic Response of a Panel Subjected to an Oscillating Flux by Reduced Order Models,” Proceedings of the 52th Structures, Structural Dynamics, and Materials Conference, Denver, Colorado, April 4-7, 2011. AIAA Paper AIAA-2011-2016.

[21] Matney, A., Perez, R., Spottswood, S.M., Wang, X.Q., and Mignolet, M.P., "Nonlinear Structural Reduced Order Modeling Methods for Hypersonic Structures”, Proceedings of the 53rd Structures, Structural Dynamics, and Materials Conference, Honolulu, Hawaii, April 2326, 2012. AIAA Paper AIAA-2012-1972.

[22] Muravyov, A.A., and Rizzi, S.A., "Determination of Nonlinear Stiffness with Application to Random Vibration of Geometrically Nonlinear Structures,” Computers and Structures, Vol. 81, pp. 1513-1523, 2003 
[23] Hollkamp, J.J., Gordon R.W., and Beberniss, T.J., "Revisiting the Nonlinear Response of a Plate to Acoustic Loading," Proceedings of the 49th Structures, Structural Dynamics, and Materials Conference, Schaumburg, Illinois, April 7-10, 2008. AIAA-2008-2233.

[24] Murthy, R., Wang, X.Q., Perez, R., Mignolet, M.P., and Richter, L.A. “Uncertainty-Based Experimental Validation of Nonlinear Reduced Order Models”, Journal of Sound and Vibration Vol. 331 (5), pp. 1097-1114, 2012.

[25] Mignolet, M.P., and Soize, C., "Stochastic Reduced Order Models for Uncertain Geometrically Nonlinear Dynamical Systems," Computer Methods in Applied Mechanics and Engineering, Vol. 197, 2008, pp. 3951-3963.

[26] Capiez-Lernout, E., Soize, C., and Mignolet, M.P., "Computational Stochastic Statics of an Uncertain Curved Structure with Geometrical Nonlinearity in Three-Dimensional Elasticity,” Computational Mechanics, Vol. 49, No. 1, pp. 87-97, 2012.

[27] Fung, Y.C., and Tong, P., Classical and Computational Solid Mechanics, World Scientific, River Edge, New Jersey, 2001.

[28] Bonet, J., and Wood, R.D., Nonlinear Continuum Mechanics for Finite Element Analysis, Cambridge University Press, Cambridge, 1997.

[29] Perez, R., Wang, X.Q., and Mignolet, M.P., "Non-Intrusive Structural Dynamic Reduced Order Modeling for Large Deformations: Enhancements for Complex Structures,” Journal of Computational and Nonlinear Dynamics, Vol. 9, No.3, 031008, 2014.

[30] Perez, R.A., "Multiscale Reduced Order Models for the Geometrically Nonlinear Response of Complex Structures,” Ph.D. Dissertation, Arizona State University, December 2012. 
Table 1. Clamped-Clamped Beam Properties

\begin{tabular}{ll}
\hline Beam Length & $0.2286 \mathrm{~m}$ \\
Cross-section Width $(w)$ & $0.0127 \mathrm{~m}$ \\
Cross-section & $7.8810-4 \mathrm{~m}$ \\
Thickness $(h)$ & $2700 \mathrm{~kg} / \mathrm{m} 3$ \\
Density & $73,000 \mathrm{MPa}$ \\
Young's Modulus & $27,730 \mathrm{MPa}$ \\
\hline
\end{tabular}

Table 2. Natural frequencies along with relative difference between notched and virgin beams.

\begin{tabular}{llll}
\hline $\begin{array}{l}\text { Mode } \\
\text { No. }\end{array}$ & $\begin{array}{l}\text { Virgin } \\
\text { Beam } \\
(\mathrm{Hz})\end{array}$ & $\begin{array}{l}\text { Notched } \\
\text { Beam } \\
(\mathrm{Hz})\end{array}$ & $\begin{array}{l}\text { Relative } \\
\text { Difference } \\
(\%)\end{array}$ \\
\hline 1 & 81.561 & 81.551 & 0.010 \\
3 & 442.075 & 441.900 & 0.040 \\
6 & 1098.527 & 1097.261 & 0.110 \\
10 & 2061.725 & 2061.693 & 0.001 \\
\hline
\end{tabular}

Table 3. Summary of prediction errors, notched beam and virgin beam ROMs.

\begin{tabular}{|c|c|c|c|c|c|c|}
\hline $\begin{array}{l}\text { Peak T3 Disp } \\
\text { (Beam } \\
\text { Thicknesses) }\end{array}$ & \multicolumn{2}{|c|}{$\begin{array}{l}\text { Prediction Error } \\
\text { Transverse (T3) } \\
\text { Component (\%) }\end{array}$} & \multicolumn{2}{|c|}{$\begin{array}{l}\text { Prediction Error } \\
\text { Transverse (T1) } \\
\text { Component (\%) }\end{array}$} & \multicolumn{2}{|c|}{$\begin{array}{l}\text { Prediction Error } \\
\text { Transverse (T2) } \\
\text { Component (\%) }\end{array}$} \\
\hline & $\begin{array}{l}\text { Notched } \\
\text { Beam }\end{array}$ & $\begin{array}{l}\text { Virgin } \\
\text { Beam }\end{array}$ & $\begin{array}{l}\text { Notched } \\
\text { Beam }\end{array}$ & $\begin{array}{l}\text { Virgin } \\
\text { Beam }\end{array}$ & $\begin{array}{l}\text { Notched } \\
\text { Beam }\end{array}$ & $\begin{array}{l}\text { Virgin } \\
\text { Beam }\end{array}$ \\
\hline 2 & 0.2 & 0.4 & 1 & 1.4 & 2.4 & 3.4 \\
\hline 4 & 0.5 & 0.9 & 3.9 & 3.7 & 5.9 & 7.4 \\
\hline-2 & 0.3 & 0.3 & 1.4 & 1.4 & 3.4 & 3 \\
\hline-4 & 1 & 0.6 & 3.8 & 3.9 & 7.4 & 7.1 \\
\hline
\end{tabular}


Table 4. Peak in-plane element stresses in the notch region for the four loading cases studied: 2.6kPa (Case 1) and 17kPa (Case 2). Relative errors ("Erel”) are with respect to the Nastran results of the notched beam.

\begin{tabular}{lcccc}
\hline \multicolumn{1}{c}{ Computation } & \multicolumn{2}{c}{ Case 1 } & \multicolumn{2}{c}{ Case 2 } \\
& MPa & Erel (\%) & MPa & Erel (\%) \\
\hline $\begin{array}{l}\text { Nastran Notched } \\
\text { Beam }\end{array}$ & 32.3 & & 105.7 & \\
$\begin{array}{l}\text { Nastran Virgin Beam } \\
+ \text { Enrichment \#1 }\end{array}$ & 32.2 & 0.3 & 107 & 1.2 \\
$\begin{array}{l}\text { Nastran Virgin Beam } \\
+ \text { Enrichment \#2 }\end{array}$ & 32.3 & 0 & 107.2 & 1.4 \\
$\begin{array}{l}\text { ROM Notched Beam } \\
\text { ROM Virgin + }\end{array}$ & 32.7 & 1.2 & 105.8 & 0.1 \\
$\begin{array}{l}\text { Enrichment \#1 } \\
\text { ROM Virgin + } \\
\text { Enrichment \#2 }\end{array}$ & 32.7 & 1.2 & 105.6 & 0.1 \\
\hline
\end{tabular}




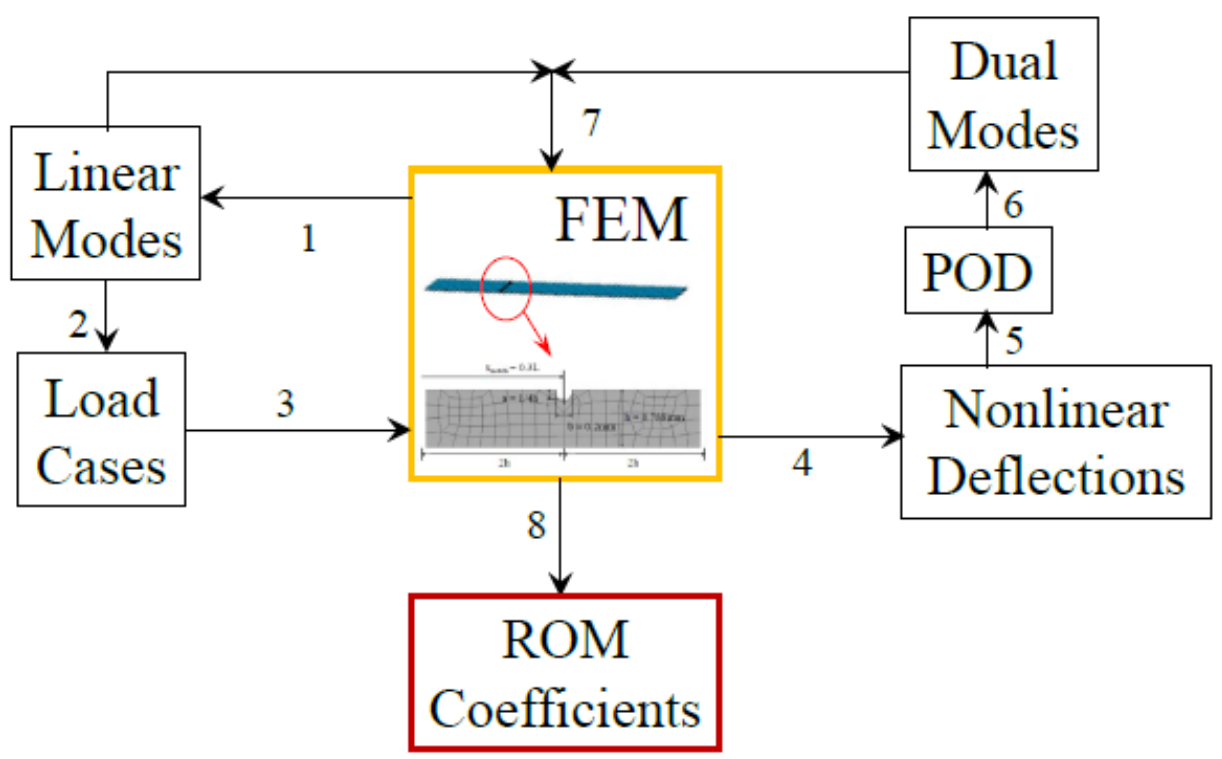

Figure 1. Overall nonlinear reduced order modeling process.

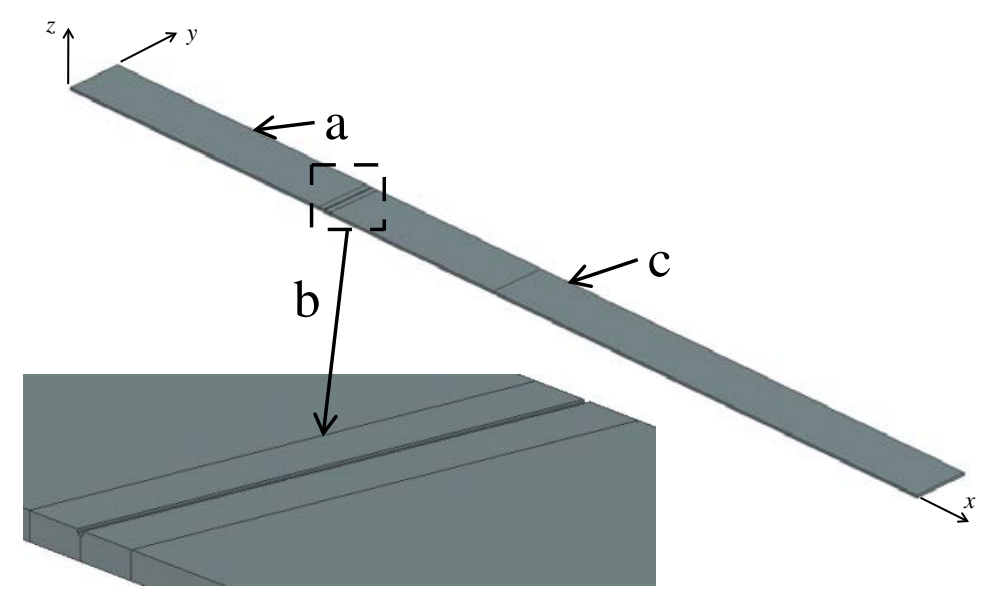

Figure 2. Notched beam model: Part (b) shows the zone near the notch with a finer mesh than parts (a) and (c) which are away from the notch.

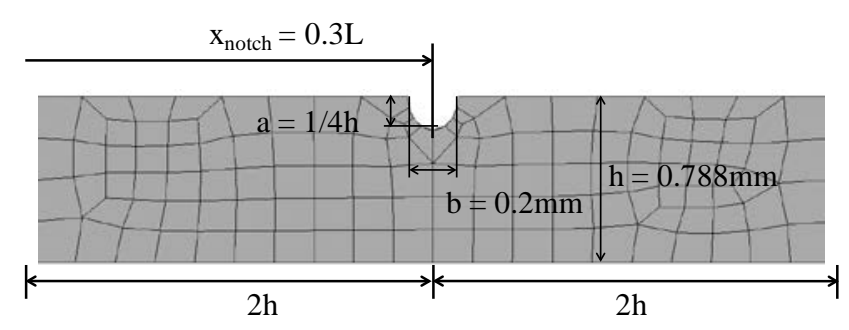

(a)

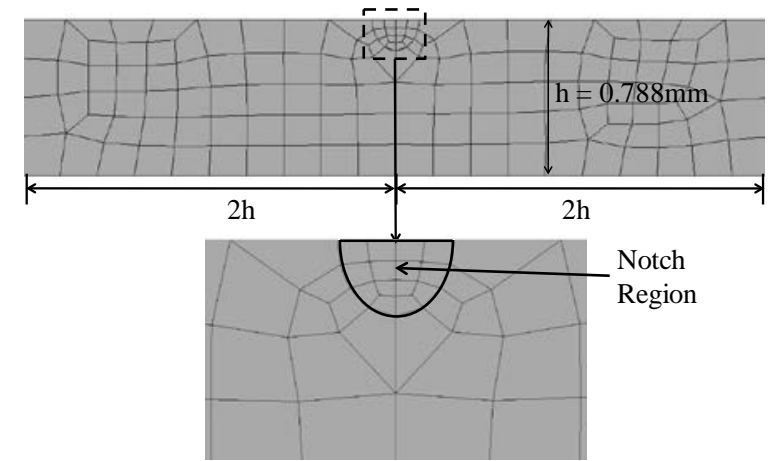

(b)

Figure 3. Geometry and finite element mesh near the notch region: (a) notched beam and (b) virgin beam. $L=0.2286 \mathrm{~m}$ and $h=7.88 \times 10-4 \mathrm{~m}$. 


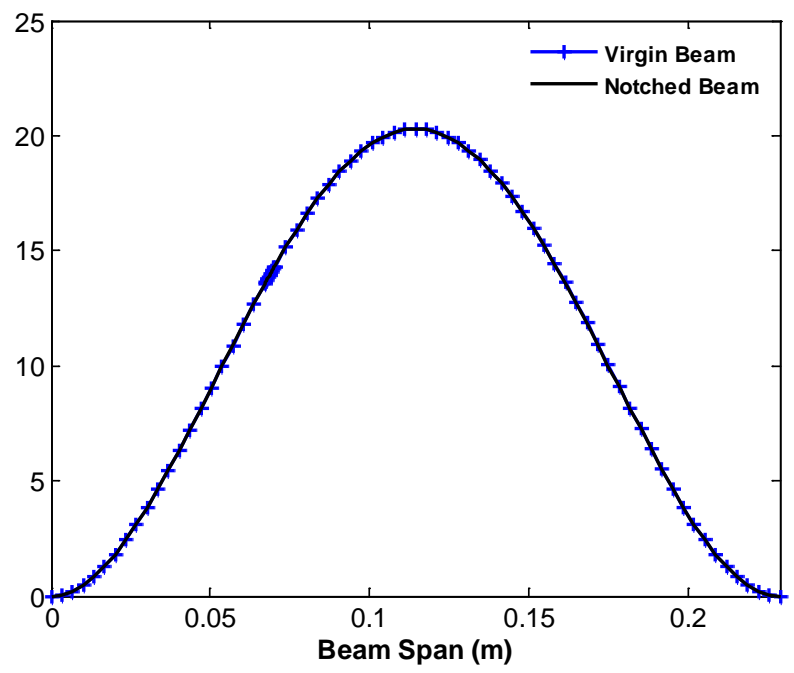

Figure 4. Transverse component of the first linear mode along one of the top edges of the beam.

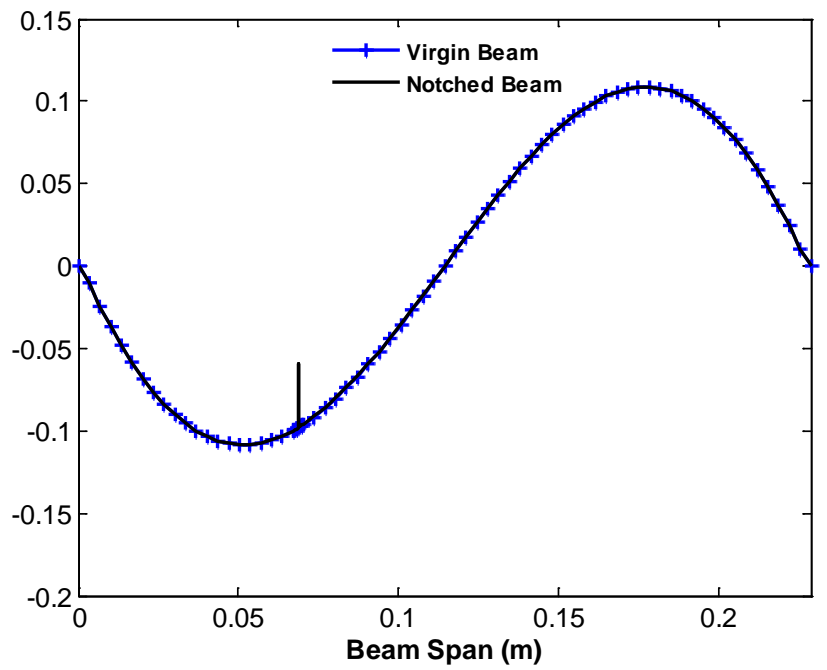

Figure 5. In-plane component of the first linear mode along one of the top edges of the beam.

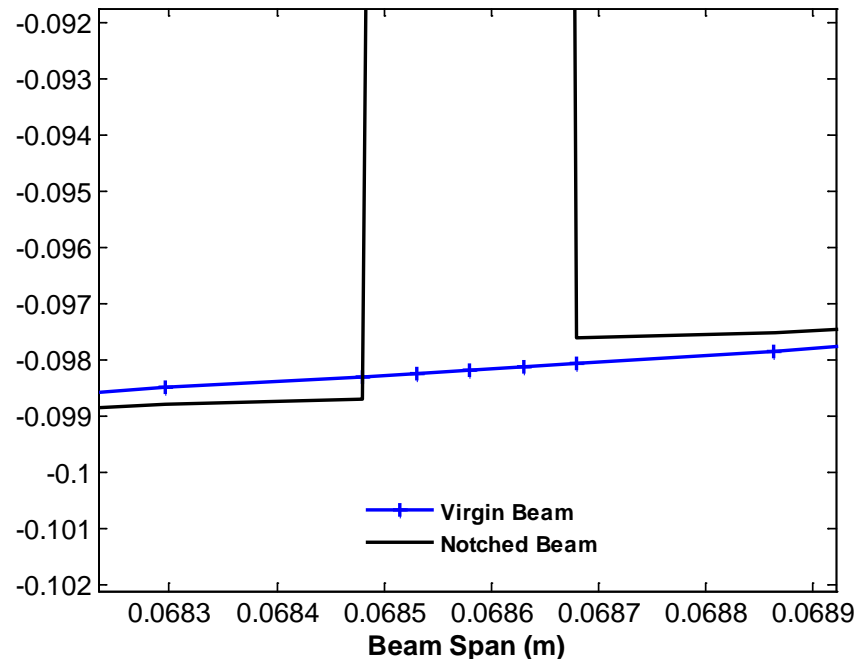

Figure 6. Zoomed-in view of the in-plane displacements near the location of the notch. 


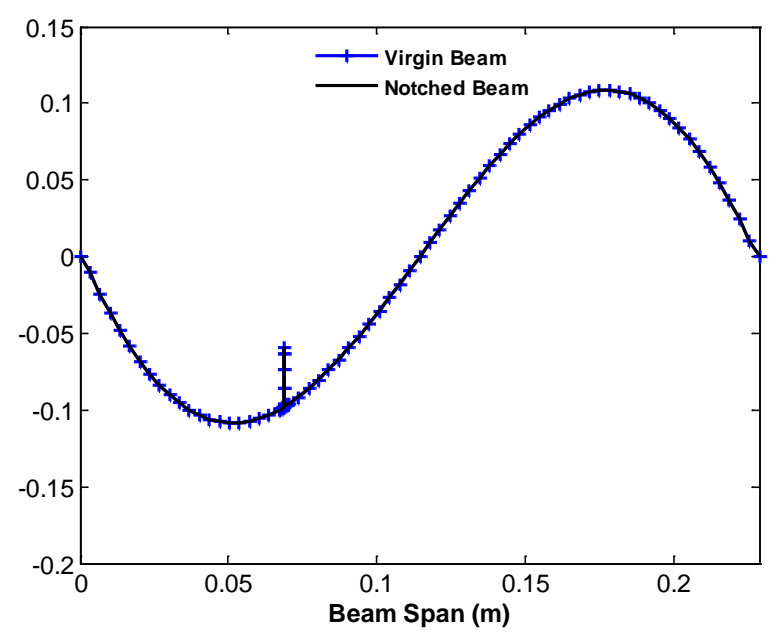

Figure 7. In-plane component of the first linear mode, curves correspond to displacements at the same nodes.

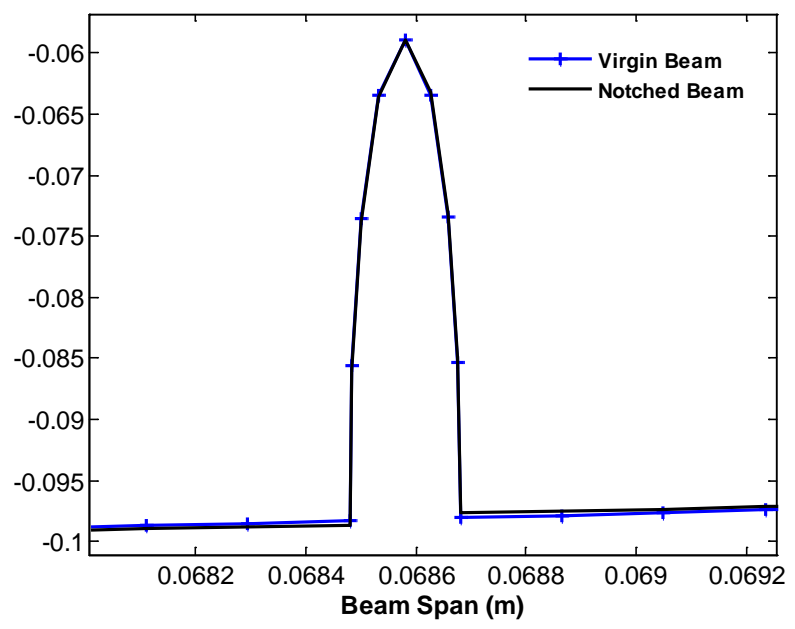

Figure 8. Zoomed-in view of the in-plane component, curves correspond to displacements at the same nodes.

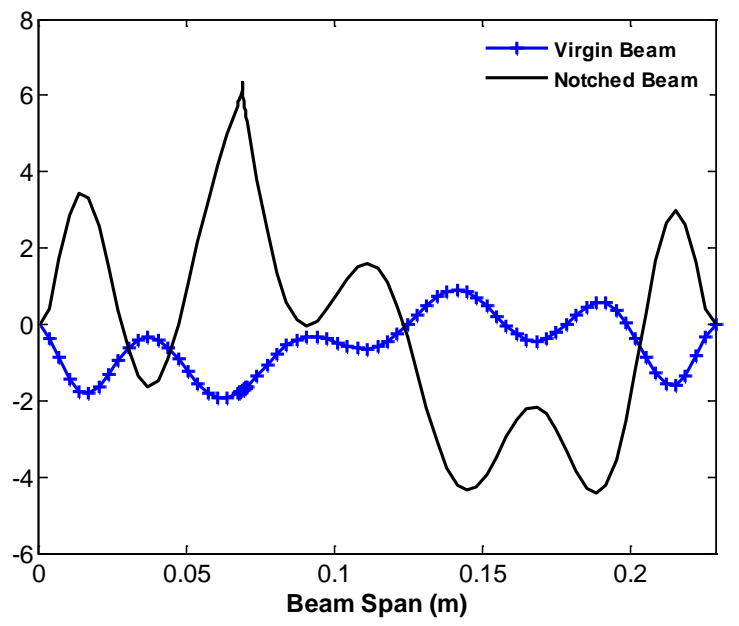

Figure 9. Transverse component of the first dual mode along one of the top edges of the beam. 


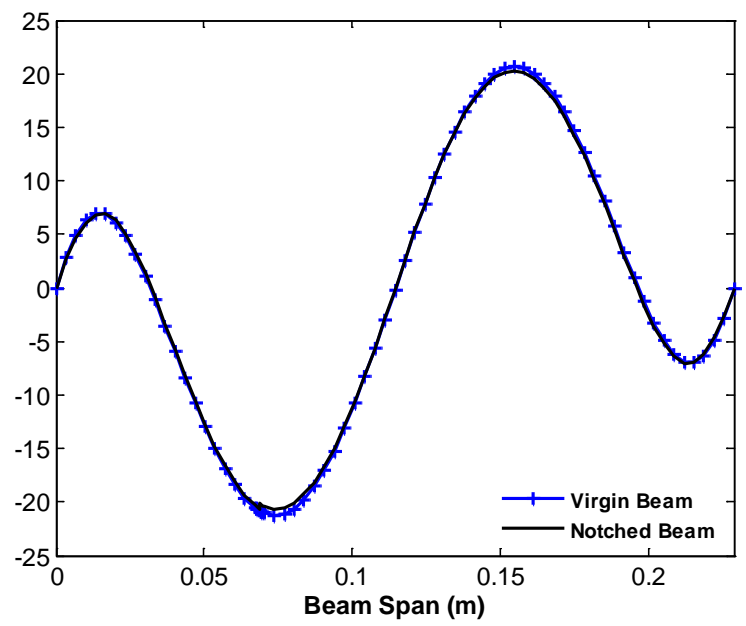

Figure 10. In-plane component of the first dual mode along one of the top edges of the beam.

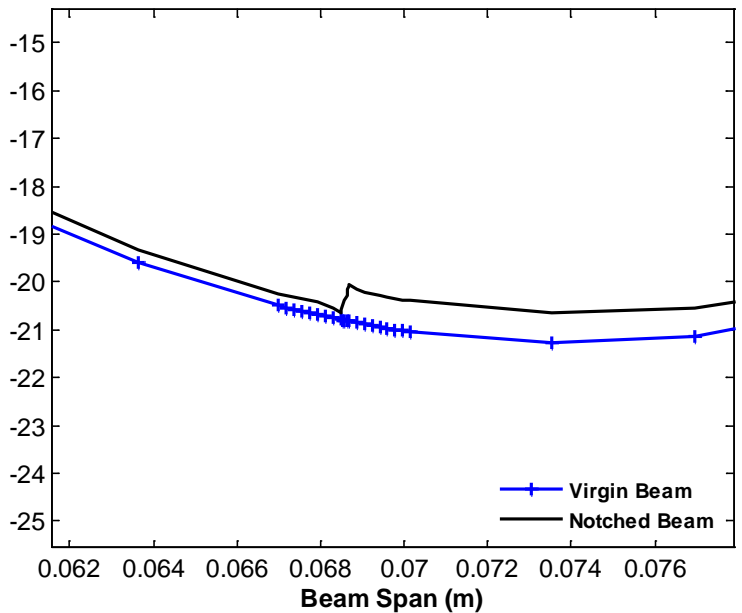

Figure 11. Zoomed-in view of the in-plane displacements near the location of the notch.

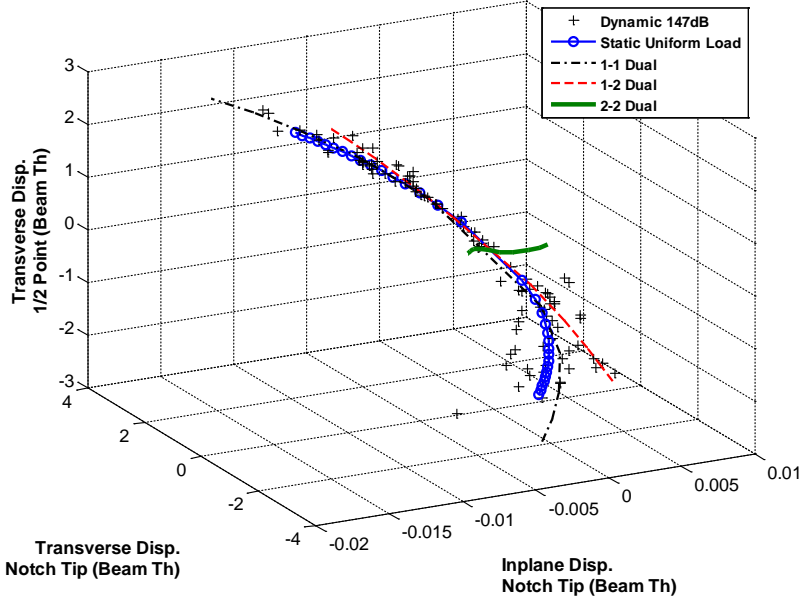

(a)

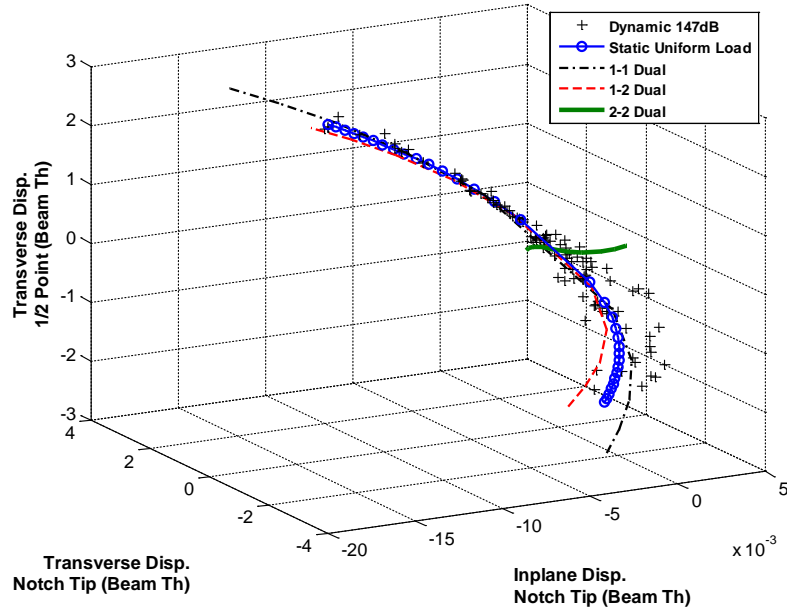

(b)

Figure 12. Displacements from Nastran at two points of a clamped-clamped beam under various loadings, transverse displacement at middle point vs. Transverse and inplane displacements at the notch tip. (a) Virgin beam. (b) Notched beam. 


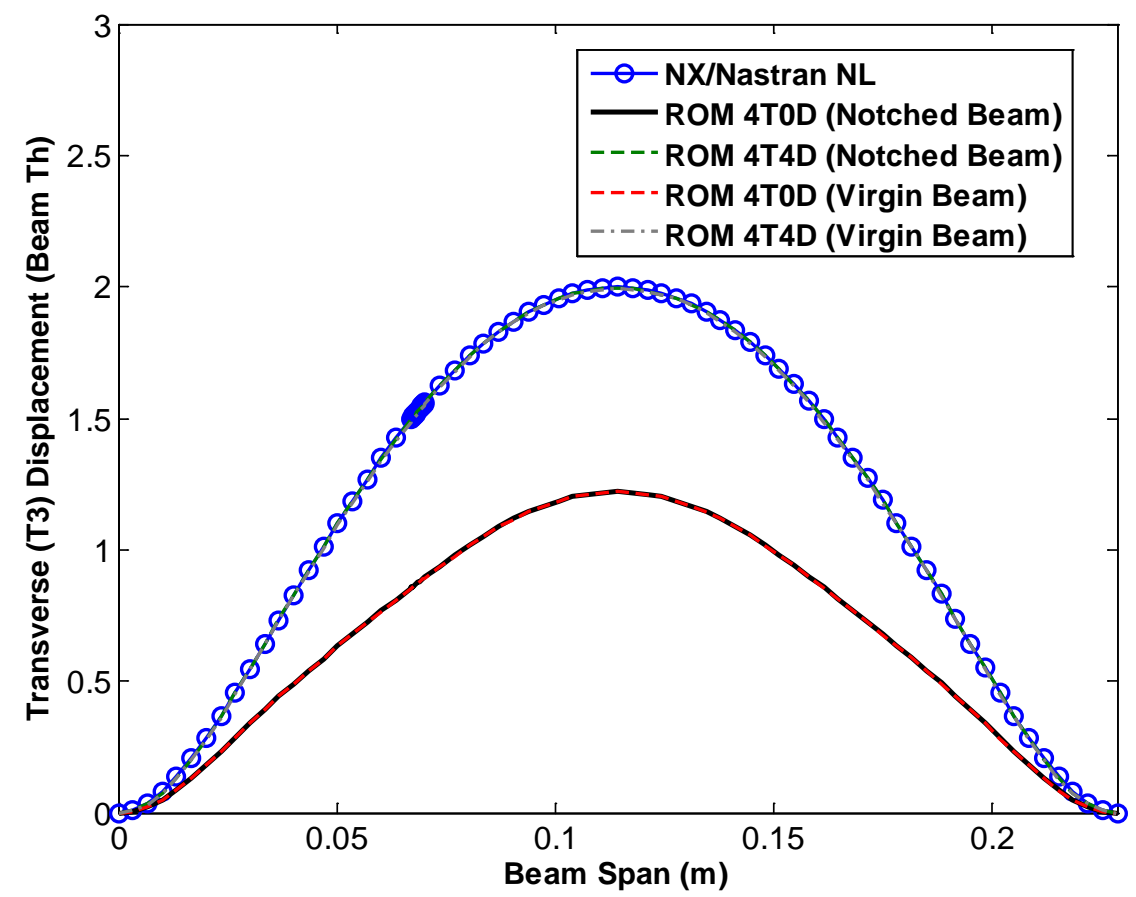

Figure 13. Transverse displacements at the top $(y=0, z=h)$ edge of the beam induced by a uniform pressure of $2.6 \mathrm{kPa}$ on its bottom surface. Reduced order models, nonlinear static FEA (“NX/Nastran NL”).

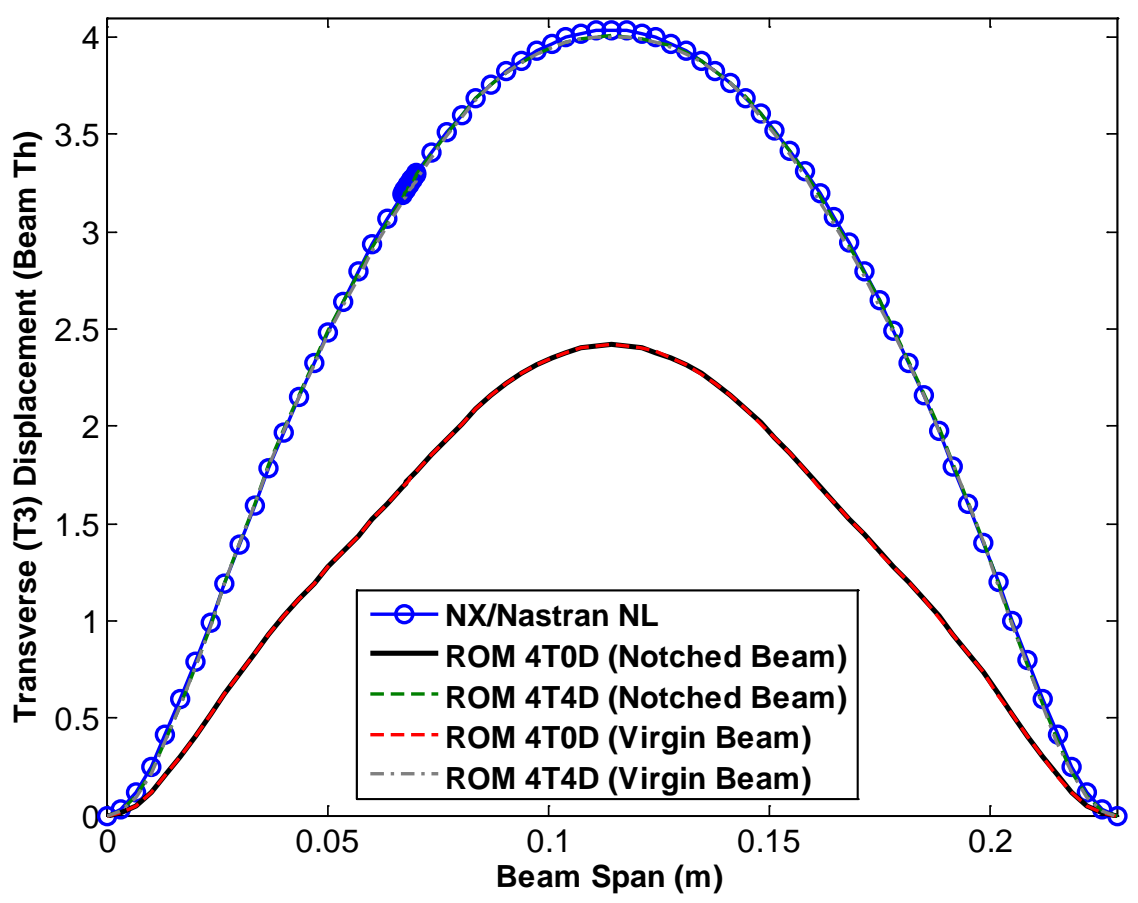

Figure 14. Transverse displacements at the top $(y=0, z=h)$ edge of the beam induced by a uniform pressure of $17 \mathrm{kPa}$ on its bottom surface. Reduced order models, nonlinear static FEA (“NX/Nastran NL”). 


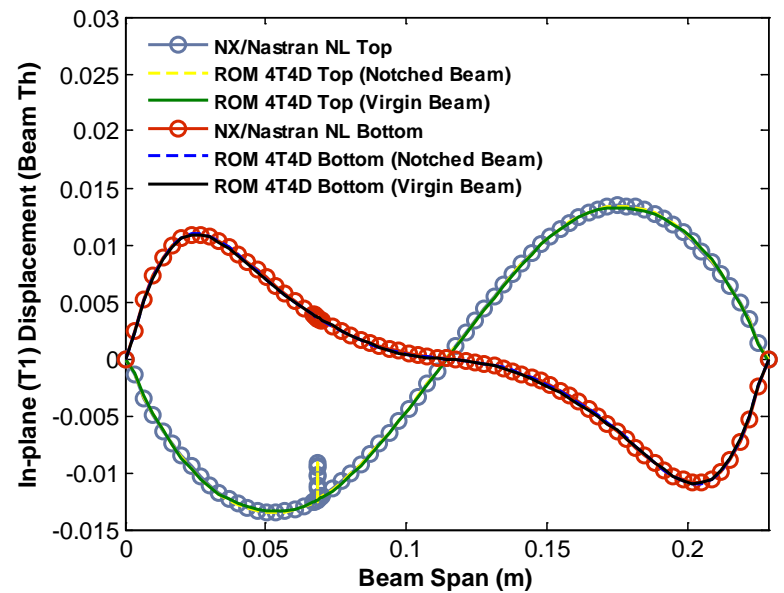

Figure 15. In-plane displacements at the top $(y=0, z=h)$ and bottom $(y=0, z=0)$ edges of the beam induced by a uniform pressure of $2.6 \mathrm{kPa}$ on its bottom surface. Reduced order models ("ROM 4T4D”), nonlinear static FEA (“NX/Nastran NL”).

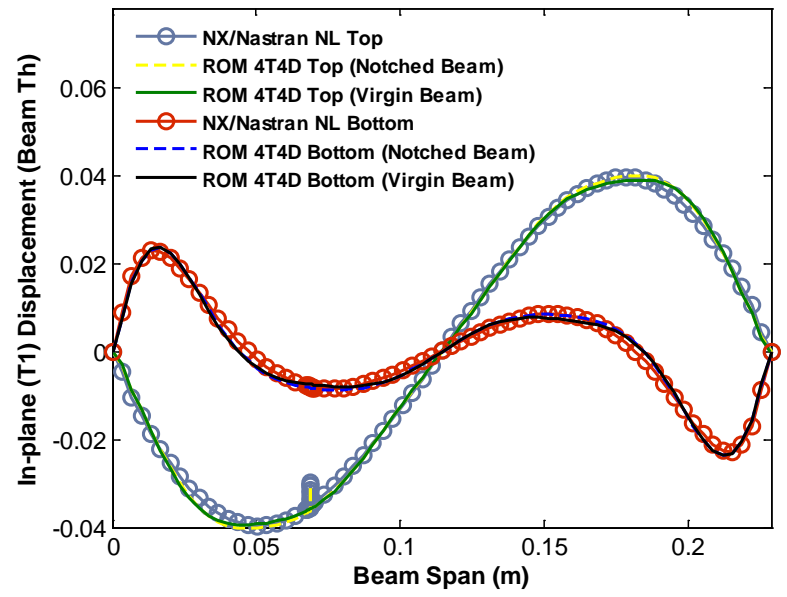

Figure 16. In-plane displacements at the top $(y=0, z=h)$ and bottom $(y=0, z=0)$ edges of the beam induced by a uniform pressure of $17 \mathrm{kPa}$ on its bottom surface. Reduced order models ("ROM 4T4D”), nonlinear static FEA (“NX/Nastran NL”). 


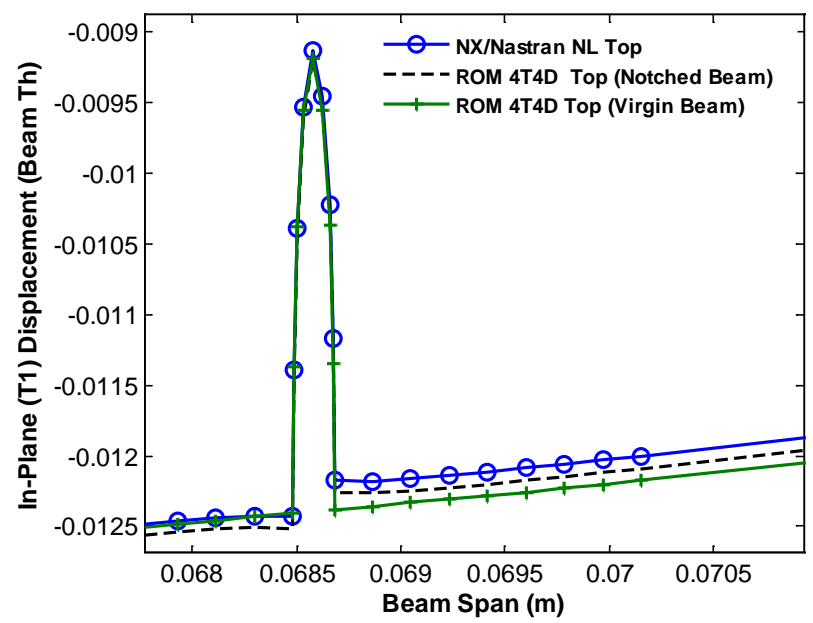

Figure 17. Close-up view of the in-plane displacements (T1) at the beam top edge $(y=0, z=h)$ due to a uniform pressure of 2.6kPa. Reduced order models ("ROM 4T4D”), nonlinear static FEA (“NX/Nastran NL”).

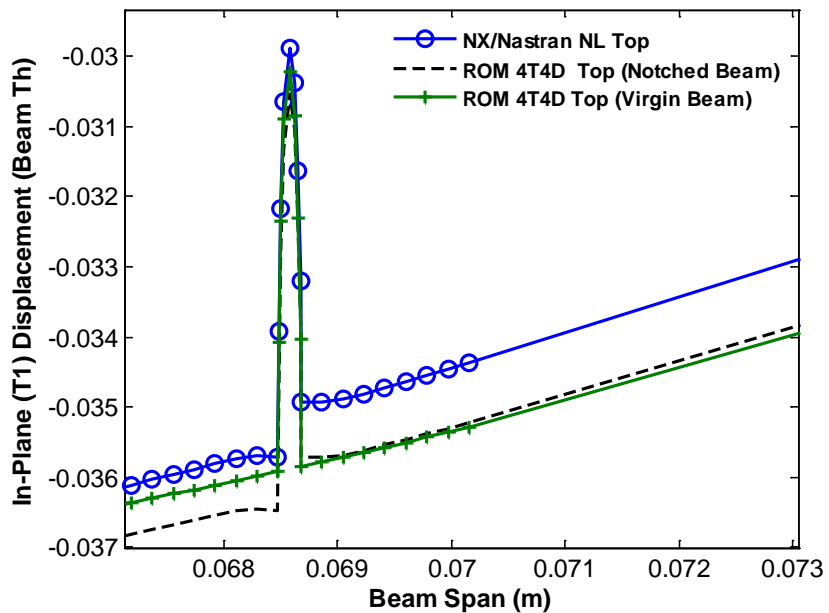

Figure 18. Close-up view of the in-plane displacements (T1) at the beam top edge $(y=0, z=h)$ due to a uniform pressure of $17 \mathrm{kPa}$. Reduced order models ("ROM 4T4D”), nonlinear static FEA (“NX/Nastran NL”). 


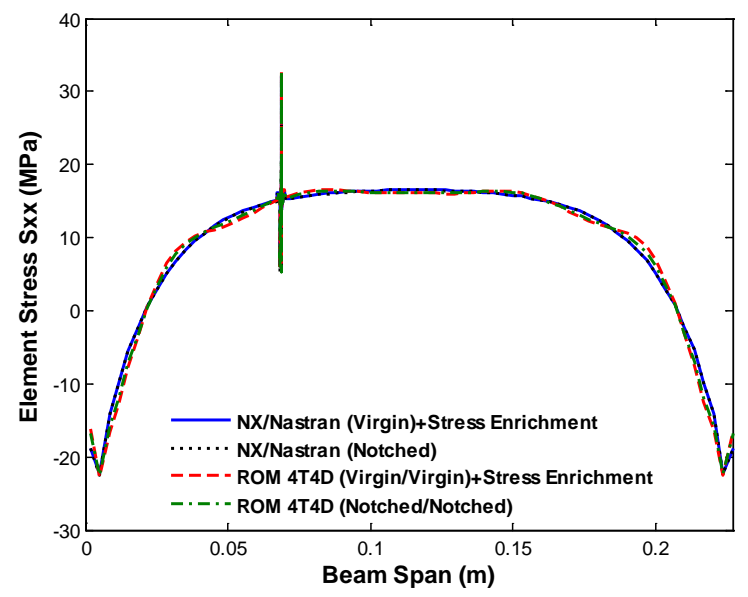

Figure 19. Element stress $S_{x x}$ near the beam edge at $y=0, z=h$, induced by a uniform pressure of 2.6kPa. Reduced order models with and without stress enrichment 1 ("ROM 4T4D") and nonlinear static FEA (“NX/Nastran”).

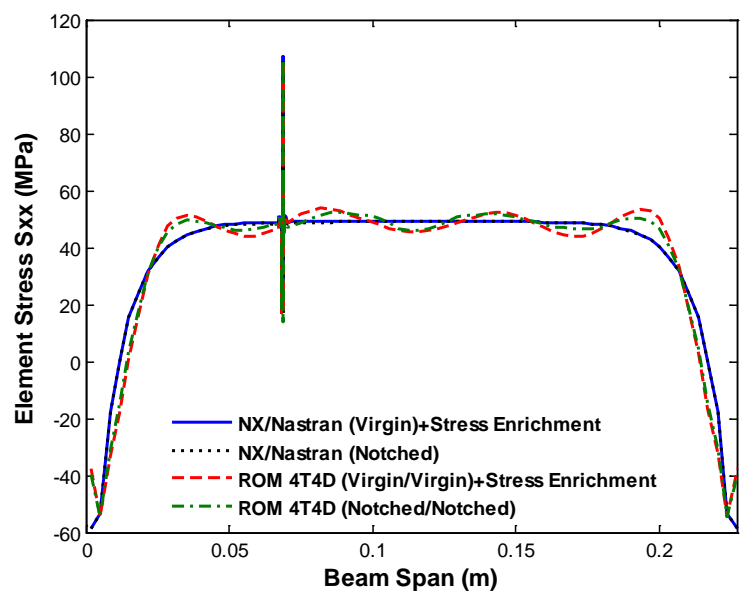

Figure 20. Element stress $S_{x x}$ near the beam edge at $y=0, z=h$, induced by a uniform pressure of $17 \mathrm{kPa}$. Reduced order models with and without stress enrichment 1 ("ROM 4T4D") and nonlinear static FEA (“NX/Nastran”). 


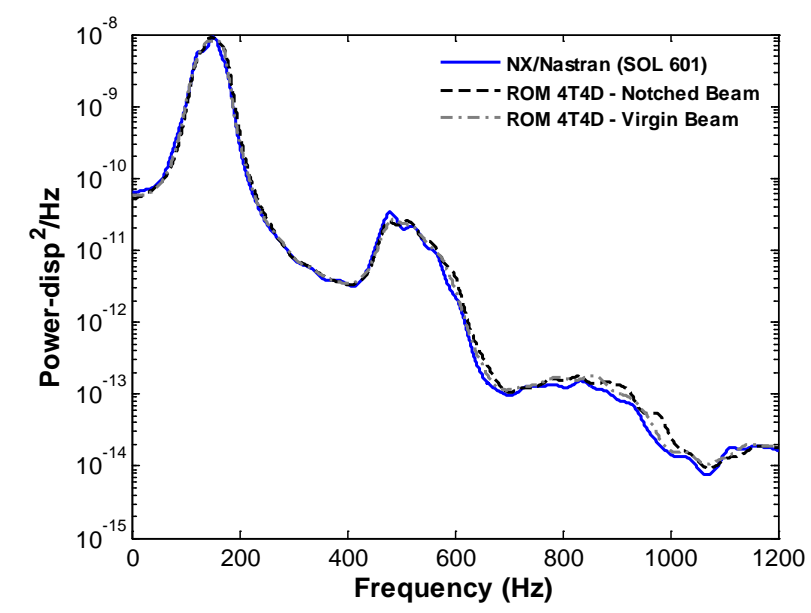

Figure 21. Power spectral density of the transverse displacement at the beam middle point, $x=L / 2, y=0, z=h(O A S P L=147 \mathrm{~dB})$. Reduced order model ("ROM(4T4D)") and FEA (“NX/Nastran”).

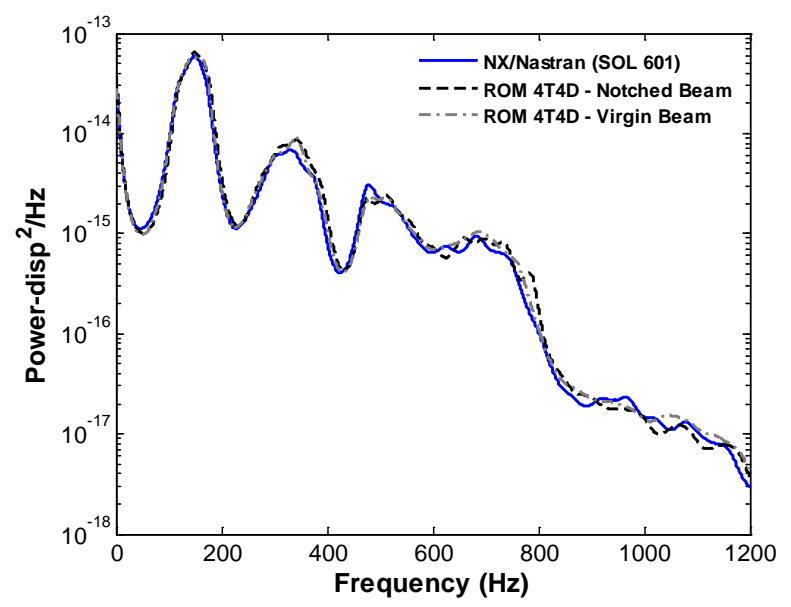

Figure 22. Power spectral density of the in-plane displacement at the notch tip $(O A S P L=$ 147dB). Reduced order model (“ROM(4T4D)") and FEA (“NX/Nastran”).

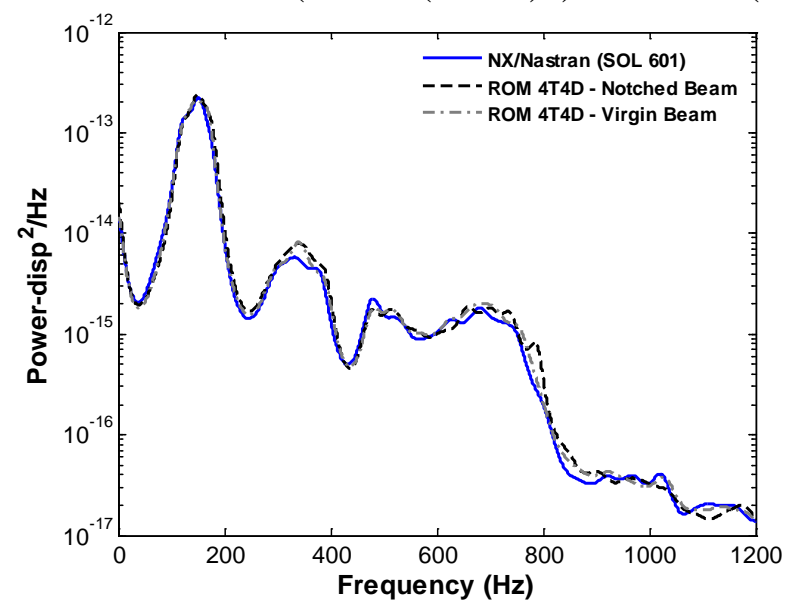

Figure 23. Power spectral density of the in-plane displacement at the beam quarter point, $x=L / 4$, $y=0, z=h(O A S P L=147 \mathrm{~dB})$. Reduced order model ("ROM(4T4D)") and FEA ("NX/Nastran"). 


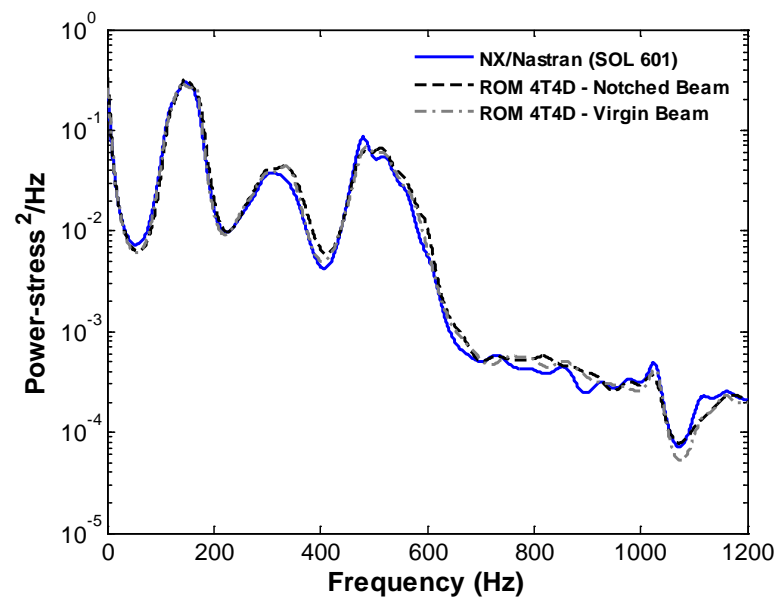

Figure 24. Power spectral density of the $S_{x x}$ element stress near the middle of the beam at $y=0$, $z=h(O A S P L=147 \mathrm{~dB})$. Reduced order model ("ROM(4T4D)") and FEA ("NX/Nastran").

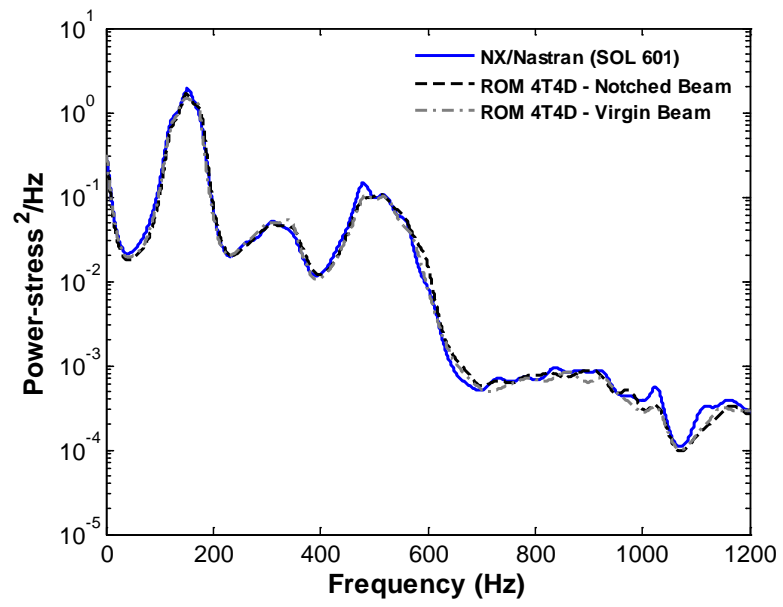

Figure 25. Power spectral density of the $S_{x x}$ element stress near the support of the beam at $y=0$, $z=h(O A S P L=147 \mathrm{~dB})$. Reduced order model ("ROM(4T4D)") and FEA ("NX/Nastran”).

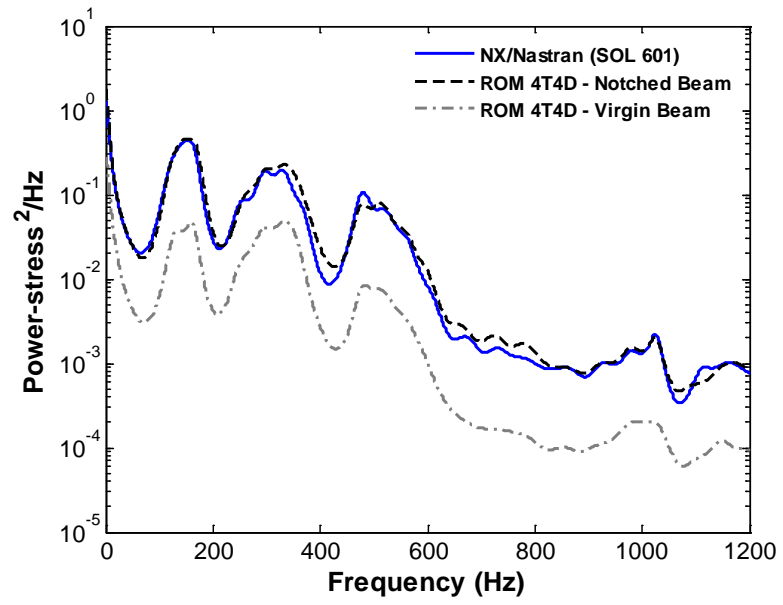

Figure 26. Power spectral density of the $S_{x x}$ element stress near the notch at $y=0, z=h(O A S P L=$ 147dB). Reduced order model (“ROM(4T4D)”) and FEA (“NX/Nastran”). 


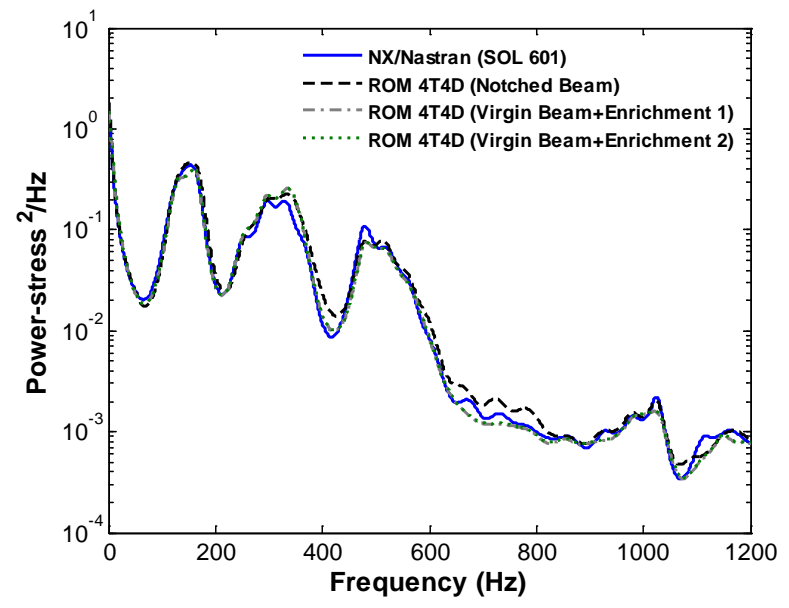

Figure 27. Power spectral density of the $S_{x x}$ element stress near the notch tip at $y=0, z=h(O A S P L$ $=147 \mathrm{~dB}$ ). Reduced order model ("ROM(4T4D)”) and FEA (“NX/Nastran”).

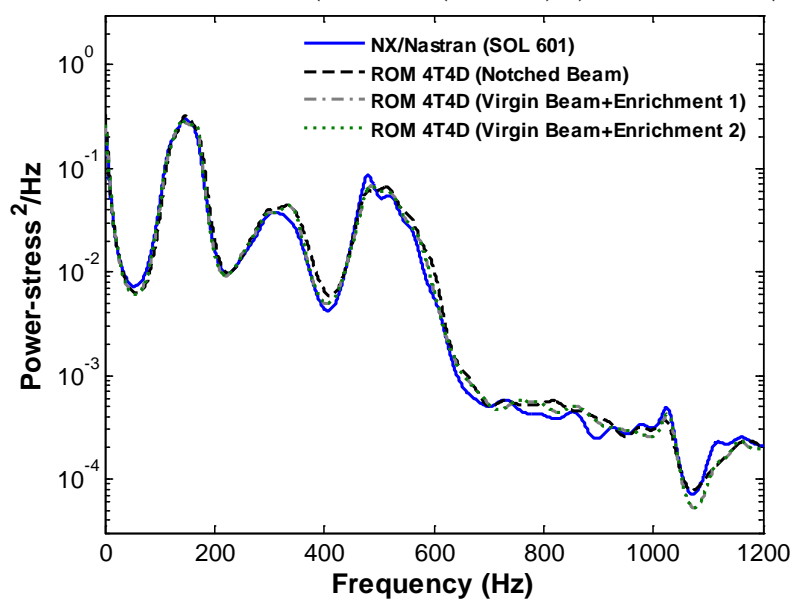

Figure 28. Power spectral density of the $S_{x x}$ element stress near the middle of the beam at $y=0$, $z=h(O A S P L=147 \mathrm{~dB})$. Reduced order model ("ROM(4T4D)”) and FEA (“NX/Nastran”).

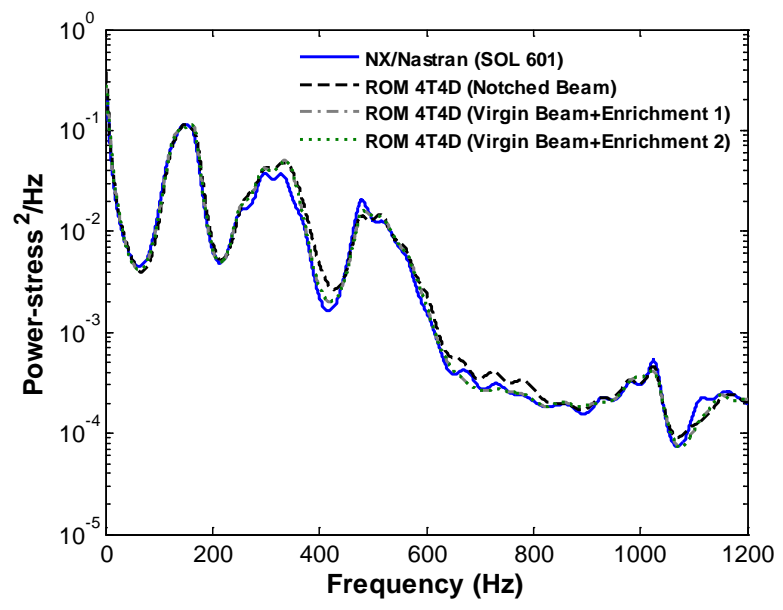

Figure 29. Power spectral density of the $S_{x x}$ element stress at 2 beam thicknesses from the notch tip and $y=0, z=h(O A S P L=147 \mathrm{~dB})$. Reduced order model ("ROM(4T4D)") and FEA

(“NX/Nastran”). 


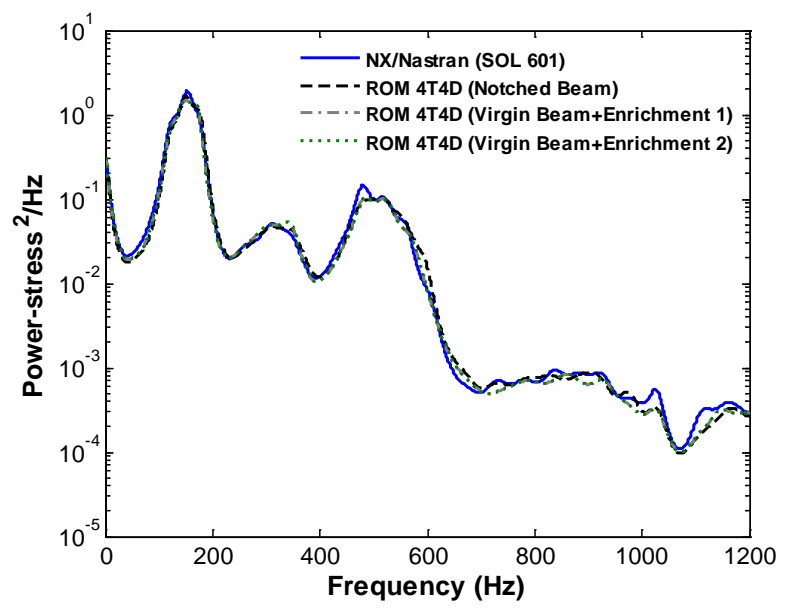

Figure 30. Power spectral density of the $S_{x x}$ element stress near the support of the beam at $y=0$, $z=h(\mathrm{OASPL}=147 \mathrm{~dB})$. Reduced order model ("ROM(4T4D)") and FEA ("NX/Nastran”).

\section{LIST OF CAPTIONS}

Figure 1. Overall nonlinear reduced order modeling process.

Figure 2. Notched beam model: Part (b) shows the zone near the notch with a finer mesh than parts (a) and (c) which are away from the notch.

Figure 3. Geometry and finite element mesh near the notch region: (a) notched beam and (b) virgin beam. Where $L=0.2286 \mathrm{~m}$ and $h=7.88 \times 10-4 \mathrm{~m}$.

Figure 4. Transverse component of the first linear mode along one of the top edges of the beam.

Figure 5. In-plane component of the first linear mode along one of the top edges of the beam.

Figure 6. Zoomed-in view of the in-plane displacements near the location of the notch.

Figure 7. In-plane component of the first linear mode, curves correspond to displacements at the same nodes.

Figure 8. Zoomed-in view of the in-plane component, curves correspond to displacements at the same nodes.

Figure 9. Transverse component of the first dual mode along one of the top edges of the beam.

Figure 10. In-plane component of the first dual mode along one of the top edges of the beam.

Figure 11. Zoomed-in view of the in-plane displacements near the location of the notch. 
Figure 12. Displacements from Nastran at two points of a clamped-clamped beam under various loadings, transverse displacement at middle point vs. Transverse and inplane displacements at the notch tip. (a) Virgin beam. (b) Notched beam.

Figure 13. Transverse displacements at the top $(y=0, z=h)$ edge of the beam induced by a uniform pressure of 2.6kPa on its bottom surface. Reduced order models (“ROM 4T4D”), nonlinear static FEA (“NX/Nastran NL”).

Figure 14. Transverse displacements at the top $(y=0, z=h)$ edge of the beam induced by a uniform pressure of $17 \mathrm{kPa}$ on its bottom surface. Reduced order models ("ROM 4T4D”), nonlinear static FEA (“NX/Nastran NL”).

Figure 15. In-plane disp. at the top $(y=0, z=h)$ and bottom $(y=0, z=0)$ edges of the beam induced by a uniform pressure of $2.6 \mathrm{kPa}$ on its bottom surface. Reduced order models ("ROM 4T4D”), nonlinear static FEA (“NX/Nastran NL”).

Figure 16. In-plane disp. at the top $(y=0, z=h)$ and bottom $(y=0, z=0)$ edges of the beam induced by a uniform pressure of $17 \mathrm{kPa}$ on its bottom surface. Reduced order models (“ROM 4T4D”), nonlinear static FEA (“NX/Nastran NL”).

Figure 17. Close-up view of the in-plane disp. (T1) at the beam top edge $(y=0, z=h)$ due to a uniform pressure of 2.6kPa. Reduced order models ("ROM 4T4D”), nonlinear static FEA

(“NX/Nastran NL”).

Figure 18. Close-up view of the in-plane disp. (T1) at the beam top edge $(y=0, z=h)$ due to a uniform pressure of 17kPa. Reduced order models ("ROM 4T4D”), nonlinear static FEA (“NX/Nastran NL”).

Figure 19. Element stress $S_{x x}$ near the beam edge at $y=0, z=h$, induced by a uniform pressure of 2.6kPa. Reduced order model with stress enrichment ("ROM 4T4D+Stress Enrichment)", nonlinear static FEA (“NX/Nastran”).

Figure 20. Element stress $S_{x x}$ near the beam edge at $y=0, z=h$, induced by a uniform pressure of $17 \mathrm{kPa}$. Reduced order model with stress enrichment ("ROM 4T4D+Stress Enrichment)", nonlinear static FEA (“NX/Nastran”).

Figure 21. Power spectral density of the transverse displacement at the beam middle point, $x=1 / 2 L, y=0, z=h(O A S P L=147 \mathrm{~dB})$. Reduced order model ("ROM(4T4D)") and FEA (“NX/Nastran”).

Figure 22. Power spectral density of the in-plane displacement at the notch tip (OASPL = 147dB). Reduced order model (“ROM(4T4D)”) and FEA (“NX/Nastran”).

Figure 23. Power spectral density of the in-plane displacement at the beam quarter point, $x=1 / 4 L$, $y=0, z=h(O A S P L=147 \mathrm{~dB})$. Reduced order model ("ROM(4T4D)") and FEA (“NX/Nastran”). 
Figure 24. Power spectral density of the $S_{x x}$ element stress near the middle of the beam at $y=0$, $z=h(O A S P L=147 \mathrm{~dB})$. Reduced order model ("ROM(4T4D)") and FEA ("NX/Nastran”).

Figure 25. Power spectral density of the $S_{x x}$ element stress near the support of the beam at $y=0$, $z=h(O A S P L=147 \mathrm{~dB})$. Reduced order model ("ROM(4T4D)") and FEA ("NX/Nastran”).

Figure 26. Power spectral density of the $S_{x x}$ element stress near the notch at $y=0, z=h(O A S P L=$ 147dB). Reduced order model (“ROM(4T4D)”) and FEA (“NX/Nastran”).

Figure 27. Power spectral density of the $S_{x x}$ element stress near the notch tip at $y=0, z=h(O A S P L$ $=147 \mathrm{~dB}$ ). Reduced order model ("ROM(4T4D)") and FEA ("NX/Nastran”).

Figure 28. Power spectral density of the $S_{x x}$ element stress near the middle of the beam at $y=0$, $z=h(O A S P L=147 \mathrm{~dB})$. Reduced order model ("ROM(4T4D)") and FEA (“NX/Nastran”).

Figure 29. Power spectral density of the $S_{x x}$ element stress at 2 beam thicknesses from the notch tip and $y=0, z=h(O A S P L=147 \mathrm{~dB})$. Reduced order model ("ROM(4T4D)") and FEA

(“NX/Nastran”).

Figure 30. Power spectral density of the $S_{x x}$ element stress near the support of the beam at $y=0$, $z=h(\mathrm{OASPL}=147 \mathrm{~dB})$. Reduced order model ("ROM(4T4D)”) and FEA (“NX/Nastran”). 